\title{
Confronting Higgs couplings from $D$-term extensions and natural SUSY at the LHC and ILC
}

\author{
Moritz McGarrie ${ }^{1,2}$, Gudrid Moortgat-Pick ${ }^{3,4}$, Stefano Porto ${ }^{3, \text { a }}$ \\ ${ }^{1}$ School of Physics and Centre for Theoretical Physics, University of the Witwatersrand, Johannesburg WITS 2050, South Africa \\ ${ }^{2}$ Faculty of Physics, Institute of Theoretical Physics, University of Warsaw, ul. Hoża 69, 00-681 Warsaw, Poland \\ ${ }^{3}$ II. Institut für Theoretische Physik, Universität Hamburg, Luruper Chaussee 149, 22761 Hamburg, Germany \\ ${ }^{4}$ DESY, Deutsches Elektronen-Synchrotron, Notkestraße 85, 22607 Hamburg, Germany
}

Received: 19 November 2014 / Accepted: 13 March 2015 / Published online: 10 April 2015

(C) The Author(s) 2015. This article is published with open access at Springerlink.com

\begin{abstract}
Non-decoupling $D$-term extensions of the MSSM enhance the tree-level Higgs mass compared to the MSSM; therefore, they relax fine-tuning and may allow lighter stops with rather low masses even without maximal mixing. We present the anatomy of various non-decoupling $D$-term extensions of the MSSM and explore the potential of the LHC and of the International Linear Collider (ILC) to determine their deviations in the Higgs couplings with respect to the Standard Model. Depending on the mass of the heavier Higgs $m_{H}$, such deviations may be constrained at the LHC and determined at the ILC. We evaluate the Higgs couplings in different models and study the prospects for a model distinction at the different stages of the ILC at $\sqrt{s}=250$, 500 and $1000 \mathrm{GeV}$, including the full luminosity upgrade and compare it with the prospects at HL-LHC.
\end{abstract}

\section{Contents}

1 Introduction . . . . . . . . . . . 1

2 A catalogue of non-decoupling $D$-terms . . . . . . 2

2.1 Minimal supersymmetric Standard Model(MSSM) 3

2.2 Vector Higgs Quiver Model . . . . . . . . . . 4

2.2.1 Higgs mass enhancement in the vector Higgs case ............ 5

2.2.2 Additional fine-tuning and the Higgs mass 7

2.2.3 Perturbative unification and the size of the

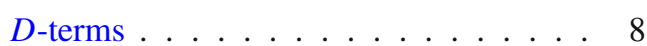

2.3 Chiral Higgs Quiver Model . . . . . . . . . . 8 2.3.1 Higgs mass enhancement in the chiral Higgs case . . . . . . . . . . 10

3 Higgs couplings determination at the LHC and ILC . 11

4 Conclusions .............. 15

a e-mail: stefano.porto@desy.de
Appendix A: General derivation of non-decoupling $D$-terms . 15 References ................ 17

\section{Introduction}

The mass of the recently discovered scalar particle $m_{h} \sim$ 125.5 GeV at the Large Hadron Collider (LHC) [1,2], as well as its measured signal strengths, within the current achievable precision, is consistent with the Higgs boson of the Standard Model (SM). In the context of Supersymmetry (SUSY), the observed Higgs mass can be obtained within the Minimal Supersymmetric Standard Model (MSSM), as well as a number of well-defined extensions of the MSSM based on the two Higgs doublet model [3]. However, having not yet observed supersymmetric particles at the LHC so far may provide circumstantial evidence that the MSSM is fine-tuned to some degree. The generation of such a heavy mass for the lightest CP-even Higgs, indeed, often requires heavy stops, posing a naturalness problem, or large stop mixings.

Motivated by the aesthetic of naturalness and in the endeavour to uniquely determine the Higgs sector and its scalar potential, in this paper we explore a number of concrete and well-motivated extensions of the MSSM and study to what degree they lead to deviations from the SM that are measurable at the LHC or at a future Higgs factory such as the International Linear Collider (ILC).

There are two main categories of extensions of the MSSM that may offer extra contributions to the Higgs mass at tree level, thereby improving fine-tuning. The first category is given by $F$-term extensions of the MSSM, in which additional fields interacting with the MSSM Higgs doublets either gauge singlets as in the NMSSM [4-9] (for a review see [10-12]) or triplets [13-17] - raise the tree-level Higgs mass via terms in the superpotential resulting in enhanced 
quartic couplings of the Higgs boson. The second category that will be studied in this work is given by quiver or $D$-term extensions of the MSSM $[18,19]$ and see also [20]. In these models, an MSSM gauge group extension provides additional non-decoupling $D$-terms from the Kähler potential, enhancing the tree-level Higgs mass through extra contributions to the Higgs quartic couplings. In particular, at a scale above the $\mathrm{TeV}$-scale, the extended gauge group under which the Higgs fields are charged is broken to $S U(2)_{L} \otimes U(1)_{Y}$; the additional $D$-terms originate from integrating out the heavier scalar fields that participate in the breaking of the gauge groups. This category of MSSM extension is appealing for a series of reasons [18, 19]: the electroweak scale remains stable after running from higher energies, as there are no log-enhanced 1-loop corrections to Higgs soft masses; additional contributions to electroweak precision observables can be suppressed and gauge coupling unification is not obviously spoiled. In addition, these models are consistent and compatible with all frameworks of supersymmetry breaking, the Higgs enhancement being largely independent of how SUSY-breaking effects are parametrised. ${ }^{1}$

We consider gauge extended MSSM models in which the gauge group features two copies of the electroweak gauge group $S U(2) \otimes U(1), G_{A}$ in site $A$ and $G_{B}$ in site $B$. At lower energies, at a scale $\gtrsim 1 \mathrm{TeV}, G_{A} \otimes G_{B}$ diagonally breaks to the SM electroweak group $S U(2)_{L} \otimes U(1)_{Y}$. In this case, two main classes of models can be identified. In the first class, which we will refer to as the "vector Higgs" case, the two Higgs doublets $H_{\mathrm{u}}$ and $H_{\mathrm{d}}$ are both charged either under $G_{A}$ or under $G_{B}$, transforming as a vector representation $\left(H_{\mathrm{u}}, H_{\mathrm{d}}\right)$ of $G_{A} \otimes G_{B}$. The second class, the "chiral Higgs" case, instead, has $H_{\mathrm{u}}$ and $H_{\mathrm{d}}$ charged under different copies of $S U(2) \otimes U(1)[21,22]$.

We supply an anatomy of these types of models, explore whether they lead to predictions that are experimentally testable at the LHC and the ILC, and we use them as a predictive guide concerning the stops masses and the trilinear $A_{\mathrm{t}}$.

The approach we take here will be bottom-up in which we neglect effects from the renormalisation group equations (RGE) and focus on these extensions as deformations of the MSSM. This approach is complementary to that of [23], for instance, where a fully UV-complete two-loop spectrum generator is used (and made publicly available, [24]) to analyse the sparticle spectrum and Higgs physics of such a quiver model. Other descriptions of quiver models as UV completions may be found, for example in [21,25-34].

\footnotetext{
1 At low energies the model is often well described by the MSSM plus an effective action. Therefore the soft terms can be parametrised largely independent of the $D$-terms enhancement, if the scale of diagonal gauge symmetry breaking is small enough.
}

The outline of this paper is as follows: in Sect. 2 we compare the minimisation conditions and naturalness between the MSSM and some of its two-site quiver extensions. In Sect. 3 we explore the LHC's and ILC's capabilities to resolve such $D$-terms enhancements of the MSSM. In Sect. 4 we present a discussion and conclude. In Appendix A we supply a more general derivation of the $D$-terms for Higgs bosons, squarks and sleptons applicable to both chiral and vector Higgs models.

\section{A catalogue of non-decoupling $D$-terms}

$D$-terms extensions of the MSSM were first explored in $[18,19]$, as they may provide a tree-level enhancement of the Higgs mass $m_{h}$ through a modification of the Higgs quartic terms in the scalar potential. A higher tree-level mass requires smaller loop-level corrections to reproduce the measured Higgs mass with respect to the MSSM, with improved consequences for naturalness. The main idea is the following: the $D$-terms induced by an extended gauge group diagonally breaking to the MSSM's $S U(2)_{L} \otimes U(1)_{Y}$ contribute to the Higgs quartic potential. The gauge symmetry breaking is caused by the acquisition of VEVs by some linking fields charged under the gauge group. The minimum of the potential is in a $D$-flat direction, leaving the Higgs doublets massless (at tree level). Once the heavy linking fields are integrated out, the associated $D$-terms do not decouple in the supersymmetric limit as soft masses for the linking fields are introduced at a scale equal or higher than the breaking scale, remaining in the Higgs scalar potential at lower energies. The additional non-decoupling $D$-terms raise the Higgs tree-level mass while introducing an effective hard SUSY breaking in the quartic scalar couplings. For more details of the generation of non-decoupling $D$-terms, see $[18,19,23]$ and Appendix A.

Non-decoupling $D$-terms extensions of MSSM may arise in two- (or more) site quiver models, for example with a single linking field $L$ between the sites, in the bifundamental representation under the two gauge group copies of $S U(2)$, as is the case in [27-30]. Alternatively, non-decoupling $D$-terms are predicted in two-site quiver models with a bifundamental and antibifundamental pair of linking fields $L, \tilde{L}[23,25,26$, $31-36]$. Furthermore, as quiver models are related to extradimensional models through deconstruction $[32,37],{ }^{2}$ nondecoupling $D$-terms may also appear in the latter context with a possible explanation of flavour hierarchies and a squark soft mass hierarchy (this was pointed out in [23]).

\footnotetext{
2 A number of models of supersymmetry breaking involve strong coupling or holography and this may be usefully approximated by holographic deconstruction [38-40], its most elementary example is given by a three-site quiver model.
} 
We wish to compare here the minimisation conditions and the implications for naturalness within the MSSM and some of its possible quiver extensions.

The gauge group of the MSSM extensions we consider is given by $G=S U(3)_{c} \otimes G_{A} \otimes G_{B}$, where $G_{A}, G_{B}$ are copies of $S U(2) \otimes U(1)$, respectively, located in sites $A$ and $B$. Regardless of how supersymmetry is broken, mediated either by gauge, gravity or some other effect, it is reasonable to approximate the low energy theory of these two-site models with the MSSM supplemented by an effective action to account for the $D$-terms. This approach neglects RGE effects, while the full implementations of the UV completions, although warranted such as in [23], are beyond the scope of this work.

\subsection{Minimal supersymmetric Standard Model (MSSM)}

It is useful, in the context of the MSSM and its $D$-term extensions, to use the most general renormalisable scalar potential for a two Higgs-doublet model (2HDM) [3],

$$
\begin{aligned}
\mathcal{V}= & m_{1}^{2}\left|H_{\mathrm{u}}\right|^{2}+m_{2}^{2}\left|H_{\mathrm{d}}\right|^{2}+m_{12}^{2}\left(H_{\mathrm{u}} H_{\mathrm{d}}+H_{\mathrm{u}}^{\dagger} H_{\mathrm{d}}^{\dagger}\right) \\
& +\frac{\lambda_{1}}{2}\left|H_{\mathrm{d}}\right|^{4}+\frac{\lambda_{2}}{2}\left|H_{\mathrm{u}}\right|^{4}+\lambda_{3}\left|H_{\mathrm{u}}\right|^{2}\left|H_{\mathrm{d}}\right|^{2} \\
& +\lambda_{4}\left|H_{\mathrm{d}}^{\dagger} H_{\mathrm{u}}\right|^{2}+\frac{\lambda_{5}}{2}\left[\left(H_{\mathrm{u}} \cdot H_{\mathrm{d}}\right)^{2}+\text { c.c. }\right] \\
& +\lambda_{6}\left|H_{\mathrm{d}}\right|^{2}\left[\left(H_{\mathrm{u}} \cdot H_{\mathrm{d}}\right)+\text { c.c. }\right] \\
& +\lambda_{7}\left|H_{\mathrm{u}}\right|^{2}\left[\left(H_{\mathrm{u}} \cdot H_{\mathrm{d}}\right)+\text { c.c. }\right],
\end{aligned}
$$

with all parameters real and CP-conserving. To recover the MSSM Higgs scalar potential, we take

$$
\begin{array}{ll}
m_{1}^{2}=\left(|\mu|^{2}+m_{H_{\mathrm{u}}}^{2}\right), \quad m_{2}^{2}=\left(|\mu|^{2}+m_{H_{\mathrm{d}}}^{2}\right), & m_{12}^{2}=B_{\mu}, \\
\lambda_{1}=\lambda_{2}=\frac{g^{2}+g^{\prime 2}}{4}, \quad-\lambda_{3}=\frac{g^{2}+g^{\prime 2}}{4}, \quad \lambda_{4}=\frac{1}{2} g^{2}, \\
\lambda_{5}=\lambda_{6}=\lambda_{7}=0,
\end{array}
$$

with $g^{\prime}, g$, respectively, being the Standard Model hypercharge and the $S U(2)_{L}$ coupling constants. ${ }^{3}$

The up- and down-Higgs doublet scalar fields may be written in terms of their charged and neutral components, $H_{\mathrm{u}}=\left(H_{\mathrm{u}}^{+}, H_{\mathrm{u}}^{0}\right), H_{\mathrm{d}}=\left(H_{\mathrm{d}}^{0}, H_{\mathrm{d}}^{-}\right)$. The minimisation conditions $\frac{\partial \mathcal{V}}{\partial H_{\mathrm{d}}^{0}}=0=\frac{\partial \mathcal{V}}{\partial H_{\mathrm{u}}^{0}}$ should be fulfilled for the consistency of the electroweak breaking minimum of the potential. The VEVs of the neutral components are defined as [41]

$\left\langle H_{\mathrm{u}}^{0}\right\rangle=\frac{v_{\mathrm{u}}}{\sqrt{2}}, \quad\left\langle H_{\mathrm{d}}^{0}\right\rangle=\frac{v_{\mathrm{d}}}{\sqrt{2}}$,

\footnotetext{
${ }^{3}$ In the following, we take $g_{1}$ to be $S U$ (5) GUT-normalised, such that $g_{1}=g_{1, \mathrm{GUT}}=\sqrt{5 / 3} g^{\prime}, g_{2}=g$ and $m_{Z}^{2}=\frac{1}{4}\left(\frac{3}{5} g_{1}^{2}+g_{2}^{2}\right)\left(v_{\mathrm{u}}^{2}+v_{\mathrm{d}}^{2}\right)$.
}

$v^{2} \equiv v_{\mathrm{u}}^{2}+v_{\mathrm{d}}^{2}=(246 \mathrm{GeV})^{2}, \quad \frac{v_{\mathrm{u}}}{v_{\mathrm{d}}} \equiv \frac{v \sin \beta}{v \cos \beta}=\tan \beta$.

The minimisation condition equations then read

$$
\begin{aligned}
& m_{H_{\mathrm{u}}}^{2}+|\mu|^{2}-B_{\mu} \cot \beta-\frac{m_{Z}^{2}}{2} \cos (2 \beta)=0, \\
& m_{H_{\mathrm{d}}}^{2}+|\mu|^{2}-B_{\mu} \tan \beta+\frac{m_{Z}^{2}}{2} \cos (2 \beta)=0,
\end{aligned}
$$

where $m_{H_{\mathrm{u}}}$ and $m_{H_{\mathrm{d}}}$ are the Higgs soft masses and $B_{\mu}$ is the MSSM $b$-term. Taking $m_{Z}^{2}$ and $\tan \beta$ as output parameters, Eqs. (2.5), (2.6) can be rewritten as

$$
\begin{aligned}
& \sin (2 \beta)=\frac{2 B_{\mu}}{m_{H_{\mathrm{u}}}^{2}+m_{H_{\mathrm{d}}}^{2}+2|\mu|^{2}}, \\
& m_{Z}^{2}=\frac{\left|m_{H_{\mathrm{d}}}^{2}-m_{H_{\mathrm{u}}}^{2}\right|}{\sqrt{1-\sin ^{2}(2 \beta)}}-m_{H_{\mathrm{u}}}^{2}-m_{H_{\mathrm{d}}}^{2}-2|\mu|^{2} .
\end{aligned}
$$

In the MSSM, after electroweak symmetry breaking there are five physical scalar states: the two CP-even neutral scalars $h$ and $H$, the CP odd neutral scalar $A^{0}$, and the conjugate charged Higgses $H^{+}, H^{-}$. Using the tree-level scalar potential minimised around the VEVs $v_{\mathrm{u}}$ and $v_{\mathrm{d}}$, one obtains the set of masses

$$
\begin{aligned}
m_{h, H}^{2, \mathrm{MSSM}}= & \frac{1}{2}\left(m_{A^{0}}^{2}+m_{Z}^{2}\right. \\
& \left.\mp \sqrt{\left(m_{A^{0}}^{2}-m_{Z}^{2}\right)^{2}+4 m_{Z^{2}}^{2} m_{A^{0}}^{2} \sin ^{2}(2 \beta)}\right), \\
m_{A^{0}}^{2, \mathrm{MSSM}} \equiv & \frac{2 B_{\mu}}{\sin 2 \beta}=2|\mu|^{2}+m_{H_{\mathrm{u}}}^{2}+m_{H_{\mathrm{d}}}^{2}, \\
m_{H^{ \pm}}^{2, \mathrm{MSSM}}= & m_{A^{0}}^{2}+m_{\mathrm{W}}^{2} .
\end{aligned}
$$

The tree-level Higgs mass is bounded by $m_{h, 0}^{2} \leq$ $m_{Z}^{2} \cos ^{2} 2 \beta$, requiring large loop corrections to reproduce the measured SM-like Higgs mass at $\sim 125.5 \mathrm{GeV}$. The MSSM Higgs mass squared in the decoupling limit $m_{A^{0}} \gg m_{Z}$ can be approximated at one loop (with two-loop leading-log effects included) by $[12,42-46]$

$$
\begin{aligned}
m_{h, 1}^{2, \mathrm{MSSM}} \simeq & m_{z}^{2} \cos ^{2} 2 \beta+\frac{3}{2 \pi^{2} v^{2}}\left[m_{t, r}^{4}\left(\sqrt{m_{t} M_{\tilde{t}}}\right) \ln \frac{M_{\tilde{t}}^{2}}{m_{t}^{2}}\right. \\
& \left.+m_{t, r}^{4}\left(M_{\tilde{t}}\right) \frac{X_{t}^{2}}{M_{\tilde{t}}^{2}}\left(1-\frac{X_{t}^{2}}{12 M_{\tilde{t}}^{2}}\right)\right]
\end{aligned}
$$

where $m_{t, r}(\Lambda)$ is the running top mass at the scale $\Lambda$ and $M_{\tilde{t}}^{2}=m_{\tilde{t}_{1}} m_{\tilde{t}_{2}} ; X_{t}=A_{t}-\mu^{*} \cot \beta$, with $A_{t}$ the stop soft SUSY-breaking trilinear coupling, quantifies stop mixing. ${ }^{4}$ This expression assumes that the left and right soft parameters of the stops are equal $m_{Q_{3}}=m_{U_{R}}$.

\footnotetext{
${ }^{4}$ In the following we assume $\mu$ to be real.
} 


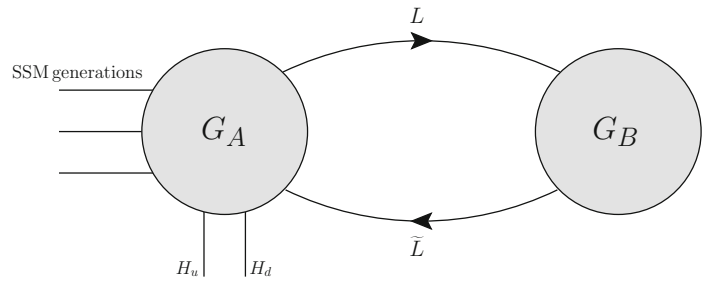

Fig. 1 The quiver module of the electroweak sector which leads to the vector-Higgs $D$-term, as in Table 1 . The supersymmetric standard model is on site $A$, the linking fields $(L, \tilde{L})$ connect the two sites. The singlet field $(K)$ is not shown. The resulting non-decoupling vectorHiggs $D$-term is displayed in Eq. (2.13)

Table 1 The matter content of the theory that may lead to a vectorHiggs non-decoupled $D$-term for both $S U(2)_{L}$ and $U(1)_{Y}$, with the Higgs doublets on site $A . f=1,2,3$ labels the generations. The singlet $\hat{K}$ couples to the linking fields in the superpotential and it is introduced to generate a suitable scalar potential for the linking fields; see also [23]. This model is represented in Fig. 1

\begin{tabular}{llll}
\hline Superfields & Spin 0 & Spin $\frac{1}{2}$ & $G_{A} \otimes G_{B} \otimes S U(3)_{c}$ \\
\hline$\hat{q}^{f}$ & $\tilde{q}^{f}$ & $q^{f}$ & $\left(\mathbf{2}, \frac{1}{6}, \mathbf{1}, 0, \mathbf{3}\right)$ \\
$\hat{d}^{f}$ & $\tilde{d}_{R}^{f *}$ & $d_{R}^{f *}$ & $\left(\mathbf{1}, \frac{1}{3}, \mathbf{1}, 0, \overline{\mathbf{3}}\right)$ \\
$\hat{u}^{f}$ & $\tilde{u}_{R}^{f *}$ & $u_{R}^{f *}$ & $\left(\mathbf{1},-\frac{2}{3}, \mathbf{1}, 0, \overline{\mathbf{3}}\right)$ \\
$\hat{l}^{f}$ & $\tilde{l}^{f}$ & $l^{f}$ & $\left(\mathbf{2},-\frac{1}{2}, \mathbf{1}, 0, \mathbf{1}\right)$ \\
$\hat{e}^{f}$ & $\tilde{e}_{R}^{f *}$ & $e_{R}^{f *}$ & $(\mathbf{1}, 1, \mathbf{1}, 0, \mathbf{1})$ \\
$\hat{H}_{\mathrm{d}}$ & $H_{\mathrm{d}}$ & $\tilde{H}_{\mathrm{d}}$ & $\left(\mathbf{2},-\frac{1}{2}, \mathbf{1}, 0, \mathbf{1}\right)$ \\
$\hat{H}_{\mathrm{u}}$ & $H_{\mathrm{u}}$ & $\tilde{H}_{\mathrm{u}}$ & $\left(\mathbf{2}, \frac{1}{2}, \mathbf{1}, 0, \mathbf{1}\right)$ \\
$\hat{L}$ & $L$ & $\psi_{L}$ & $\left(\mathbf{2},-\frac{1}{2}, \overline{\mathbf{2}}, \frac{1}{2}, \mathbf{1}\right)$ \\
$\hat{\tilde{L}}$ & $\tilde{L}$ & $\psi_{\tilde{L}}$ & $\left(\overline{\mathbf{2}}, \frac{1}{2}, \mathbf{2},-\frac{1}{2}, \mathbf{1}\right)$ \\
$\hat{K}$ & $K$ & $\psi_{K}$ & $(\mathbf{1}, 0, \mathbf{1}, 0, \mathbf{1})$ \\
\hline
\end{tabular}

\subsection{Vector Higgs Quiver Model}

The first class of two-sites quiver models that we consider is given by the "vector Higgs" case, in which both Higgs doublets of the MSSM are on the same site $[18,19]$. The particular scenario in which the Higgs doublets as well as the other MSSM matter fields are on site $A$, i.e. charged under $G_{A}$, is depicted in Fig. 1, as described in Table 1 .

As outlined at the beginning of the section, after the symmetry breaking of $G_{A} \otimes G_{B}$ to $S U(2)_{L} \otimes U(1)_{Y}$, the real uneaten scalar components of the linking fields appear in both the $A$ and the $B$ site scalar $D$-term potential. When these components are integrated out, in the effective theory the following relevant terms are added to the MSSM Higgs potential:

$$
\begin{aligned}
\delta \mathcal{L}= & -\frac{3}{5} \frac{g_{1}^{2} \Delta_{1}}{8}\left(H_{\mathrm{u}}^{\dagger} H_{\mathrm{u}}-H_{\mathrm{d}}^{\dagger} H_{\mathrm{d}}\right)^{2} \\
& -\frac{g_{2}^{2} \Delta_{2}}{8} \sum_{a}\left(H_{\mathrm{u}}^{\dagger} \sigma^{a} H_{\mathrm{u}}+H_{\mathrm{d}}^{\dagger} \sigma^{a} H_{\mathrm{d}}\right)^{2}+\cdots
\end{aligned}
$$

The ellipsis denote terms involving other scalars of the model as explained in Appendix A. $\Delta_{1}$ and $\Delta_{2}$ are respectively given by

$\Delta_{1}^{A}=\left(\frac{g_{A 1}^{2}}{g_{B 1}^{2}}\right) \frac{m_{L}^{2}}{m_{v 1}^{2}+m_{L}^{2}}, \quad \Delta_{2}^{A}=\left(\frac{g_{A 2}^{2}}{g_{B 2}^{2}}\right) \frac{m_{L}^{2}}{m_{v 2}^{2}+m_{L}^{2}}$,

where $g_{A 1}, g_{B 1}$ are the $U(1)$ couplings on site $A$ and $B$ while $g_{A 2}, g_{B 2}$ are the $S U(2)$ couplings; $m_{L}$ is the soft mass, which we assume equal for both linking fields $L, \tilde{L}$, and $m_{v_{1}}, m_{v_{2}}$ are the masses of the heavy gauge bosons after the symmetry breaking to $S U(2)_{L} \otimes U(1)_{Y}$. The relation between the MSSM gauge couplings and that of the extended gauge groups takes the form

$$
\cos \theta_{i}=\frac{g_{i}}{g_{A i}}, \quad \sin \theta_{i}=\frac{g_{i}}{g_{B i}} .
$$

To enhance the $D$-terms one requires $g_{A i}^{2}>g_{B i}^{2}$, a condition that in some cases can be problematic for perturbative unification, because, if most of the matter is charged under $G_{A}$, then a Landau pole may be reached below the GUT scale (see Sect. 2.2.3). If we are not concerned by coupling unification, then $\Delta_{1}$ and $\Delta_{2}$ may arise independently and in general are not equal in value.

For the vector Higgs extension of the MSSM, the minimisation conditions are given by

$$
\begin{aligned}
& m_{H_{\mathrm{u}}}^{2}+|\mu|^{2}-B_{\mu} \cot \beta-\frac{m_{Z}^{2}+m_{\Delta}^{2}}{2} \cos (2 \beta)=0, \\
& m_{H_{\mathrm{d}}}^{2}+|\mu|^{2}-B_{\mu} \tan \beta+\frac{m_{Z}^{2}+m_{\Delta}^{2}}{2} \cos (2 \beta)=0,
\end{aligned}
$$

where we defined $4 m_{\Delta}^{2}=\left(\frac{3}{5} g_{1}^{2} \Delta_{1}+g_{2}^{2} \Delta_{2}\right) v^{2}$. Equations (2.16), (2.17) solved for $m_{Z}^{2}$ and $\tan \beta$ read

$$
\begin{aligned}
& \sin (2 \beta)=\frac{2 B_{\mu}}{m_{H_{\mathrm{u}}}^{2}+m_{H_{\mathrm{d}}}^{2}+2|\mu|^{2}}, \\
& m_{Z}^{2}+m_{\Delta}^{2}=\frac{\left|m_{H_{\mathrm{d}}}^{2}-m_{H_{\mathrm{u}}}^{2}\right|}{\sqrt{1-\sin ^{2}(2 \beta)}}-m_{H_{\mathrm{u}}}^{2}-m_{H_{\mathrm{d}}}^{2}-2|\mu|^{2} .
\end{aligned}
$$

The tree-level Higgs masses are found simply by replacing $m_{Z}^{2} \rightarrow m_{Z}^{2}+m_{\Delta}^{2}$ and $m_{\mathrm{W}}^{2} \rightarrow m_{\mathrm{W}}^{2}\left(1+\Delta_{2}\right)$ in (2.9)-(2.11). We report here for completeness the Higgs mass matrices, and for brevity we set

$$
\begin{aligned}
& g_{12}^{2}=\frac{3}{5} g_{1}^{2}\left(1+\Delta_{1}^{2}\right)+g_{2}^{2}\left(1+\Delta_{2}^{2}\right), \\
& \hat{g}_{12}^{2}=-\frac{3}{5} g_{1}^{2}\left(1+\Delta_{1}^{2}\right)+g_{2}^{2}\left(1+\Delta_{2}^{2}\right) .
\end{aligned}
$$

The mass matrix for the CP-even Higgses, in the basis of the real components of $\left(H_{\mathrm{d}}^{0}, H_{\mathrm{u}}^{0}\right)$ is given by 
$m_{h}^{2}=\left(\begin{array}{cc}m_{h, 11} & -\frac{1}{4} g_{12}^{2} v_{\mathrm{d}} v_{\mathrm{u}}-\operatorname{Re}\left[B_{\mu}\right] \\ -\frac{1}{4} g_{12}^{2} v_{\mathrm{d}} v_{\mathrm{u}}-\operatorname{Re}\left[B_{\mu}\right] & m_{h, 22}\end{array}\right)$,

where

$m_{h, 11}=\frac{1}{8}\left(8 m_{H_{\mathrm{d}}}^{2}+8|\mu|^{2}+g_{12}^{2}\left(3 v_{d}^{2}-v_{u}^{2}\right)\right)$,

$m_{h, 22}=\frac{1}{8}\left(8 m_{H_{\mathrm{u}}}^{2}+8|\mu|^{2}-g_{12}^{2}\left(-3 v_{u}^{2}+v_{d}^{2}\right)\right)$.

For the pseudo-scalar Higgses, the mass matrix in the basis of the imaginary components of $\left(H_{\mathrm{d}}^{0}, H_{\mathrm{u}}^{0}\right)$ reads

$m_{A^{0}}^{2}=\left(\begin{array}{ll}m_{A^{0}, 11} & \operatorname{Re}\left[B_{\mu}\right] \\ \operatorname{Re}\left[B_{\mu}\right] & m_{A^{0}, 22}\end{array}\right)$,

where

$m_{A^{0}, 11}=\frac{1}{8}\left(8 m_{H_{\mathrm{d}}}^{2}+8|\mu|^{2}+g_{12}^{2}\left(-v_{u}^{2}+v_{d}^{2}\right)\right)$,

$m_{A^{0}, 22}=\frac{1}{8}\left(8 m_{H_{\mathrm{u}}}^{2}+8|\mu|^{2}-g_{12}^{2}\left(-v_{u}^{2}+v_{d}^{2}\right)\right)$.

The mass matrix for the charged Higgses $\left(H_{\mathrm{d}}^{-}, H_{\mathrm{u}}^{+, *}\right)$, $\left(H_{\mathrm{d}}^{-, *}, H_{\mathrm{u}}^{+}\right)$reads

$m_{H^{-}}^{2}=\left(\begin{array}{cc}m_{H^{-}, 11} & \frac{1}{4}\left(4 B_{\mu}^{*}+\left(g_{2}^{2}+g_{2}^{2} \Delta_{2}^{2}\right) v_{\mathrm{d}} v_{\mathrm{u}}\right) \\ \frac{1}{4}\left(4 B_{\mu}+\left(g_{2}^{2}+g_{2}^{2} \Delta_{2}^{2}\right) v_{\mathrm{d}} v_{\mathrm{u}}\right) & m_{H^{-}, 22}\end{array}\right)$,

with

$m_{H^{-}, 11}=\frac{1}{8}\left(8 m_{H_{\mathrm{d}}}^{2}+8|\mu|^{2}+g_{12}^{2} v_{d}^{2}+\hat{g}_{12}^{2} v_{u}^{2}\right)$,

$m_{H^{-}, 22}=\frac{1}{8}\left(8 m_{H_{\mathrm{u}}}^{2}+8|\mu|^{2}+g_{12}^{2} v_{u}^{2}+\hat{g}_{12}^{2} v_{d}^{2}\right)$.

The non-decoupling $D$-terms contribution causes a shift in the tree-level Higgs mass squared $m_{h, 0}^{2}$ which in the decoupling limit results in

$m_{h, 0}^{2, \mathrm{vec}}=\left[m_{Z}^{2}+\left(\frac{\frac{3}{5} g_{1}^{2} \Delta_{1}+g_{2}^{2} \Delta_{2}}{4}\right) v^{2}\right] \cos ^{2} 2 \beta$.

Also the masses of the other scalar of the model, the sfermions, are affected by additional $D$-terms; see Appendix A. For the analysed case with matter as in Table 1, the mixing matrix $M_{\tilde{f}}$ of a generic sfermion $\tilde{f}$ is given by

$M_{\tilde{f}}^{2}=\left(\begin{array}{cc}m_{\tilde{f}_{L}}^{2}+m_{f}^{2}+\hat{M}_{Z}^{2}\left(I_{3}^{f}-Q_{f} s_{\mathrm{W}}^{2}\right) & m_{f} X_{f}^{*} \\ m_{f} X_{f} & m_{\tilde{f}_{R}}^{2}+m_{f}^{2}+\hat{M}_{Z}^{2} Q_{f} s_{\mathrm{W}}^{2}\end{array}\right)$,

denoting $s_{w}=\sin \theta_{\mathrm{W}}$ where $\theta_{\mathrm{W}}$ is the Weinberg weak mixing angle, and the useful abbreviation $\hat{M}_{Z}^{2} \equiv\left(m_{Z}^{2}+m_{\Delta}^{2}\right) \cos 2 \beta$, where $m_{\Delta}^{2}=\frac{1}{2}\left(\frac{3}{5} g_{1}^{2} \Delta_{1}+g_{2}^{2} \Delta_{2}\right) v^{2}$. The off-diagonal element
$X_{f}$ is defined in terms of the soft SUSY-breaking trilinear coupling $A_{f}$ via

$X_{f}=A_{f}-\mu^{*}\{\cot \beta, \tan \beta\}$,

where $\cot \beta$ applies for the up-type quarks, $f=u, c, t$, and $\tan \beta$ applies for the down-type fermions, $f=d, s, b, e, \mu, \tau$. Note that $m_{f}, Q_{f}$ and $I_{3}^{f}$ are the mass, charge and isospin projection of the fermion $f$, respectively. Once diagonalised this matrix leads to the light and heavy sfermion masses $m \tilde{f}_{1}$ and $m_{\tilde{f}_{2}}$. In particular the stop masses are given by

$$
\begin{aligned}
m_{\tilde{t}_{1,2}}^{2} & =m_{t}^{2}+\frac{1}{2}\left[M_{\tilde{Q}_{3}}^{2}+M_{U_{3}}^{2}+\frac{1}{2} \hat{M}_{Z}^{2} \cos 2 \beta\right. \\
\mp & \sqrt{\left.\left[M_{Q_{3}}^{2}-M_{U_{3}}^{2}+\hat{M}_{Z}^{2} \cos 2 \beta\left(\frac{1}{2}-\frac{4}{3} \sin ^{2} \theta_{\mathrm{W}}\right)\right]^{2}+4 m_{t}^{2} X_{t}^{2}\right]},
\end{aligned}
$$

writing $m_{\tilde{t}_{L}}=M_{Q_{3}}, m_{\tilde{t}_{R}}=M_{U_{3}}$. To obtain the MSSM mass expression one has just to set $m_{\Delta}=0$. For a light stop scenario, this may have an appreciable effect and similarly, for the stau which may be the NLSP (for a Goldstino LSP scenario such as GMSB). In the case that the $g_{A}>g_{B}$, even for the case of split families the above mixing matrix will still apply to the third generation scalars on Site $A$. For the sneutrinos the mass matrix is given by

$m_{\tilde{\nu}}^{2}=M_{L}^{2}+\frac{1}{2}\left(m_{Z}^{2}+m_{\Delta}^{2}\right) \cos (2 \beta)$.

\subsubsection{Higgs mass enhancement in the vector Higgs case}

In the following discussion of the impact of non-decoupling $D$-terms in the vector Higgs case on the Higgs mass, we take for simplicity $\Delta_{1}=\Delta_{2} \equiv \Delta$. The effect of the tree-level shift of the Higgs mass can significantly reduce fine-tuning in the top-stop sector and allows for a reduced average stop mass. This can be seen in Fig. 2 (similarly to [36]), where we plot in the $\left(m_{Q_{3}}, X_{t}\right)$ and $\left(m_{\tilde{t}_{1}}, X_{t}\right)$ planes the Higgs mass from Eq. (2.12) with the tree-level $D$-terms corrections from Eq. (2.30), for different values of $\Delta$. While at the MSSM limit $\Delta=0$, for $X_{t}=0 \mathrm{GeV}$, we need $m_{\tilde{t}_{1}} \simeq 4 \mathrm{TeV}$ to reproduce the correct Higgs, at $\Delta=0.3$ this is possible with $m_{\tilde{t}_{1}} \simeq 1$ $\mathrm{TeV}$. One can also note that the value of the maximal mixing scenario (the sharply acute concave kink in the contours for $\left|A_{t}\right| \simeq \sqrt{6} M_{S}$ ) can further allow for a significantly smaller $X_{t}$ for increasing $\Delta$.

Discussing the expected order of the size of these $D$ terms one can observe that with $\Delta \sim \mathcal{O}(1)$ the tree-level Higgs mass would already be sufficiently large to account for the observed 125.5 GeV Higgs mass. In [36] was shown that demanding fine-tuning no worse than 1/10 together with light stops one would expect $\Delta \gtrsim 0.5$. On the other hand, in [23] it was found that $\mathcal{O}(0.1) \Delta$ is more easily obtainable 

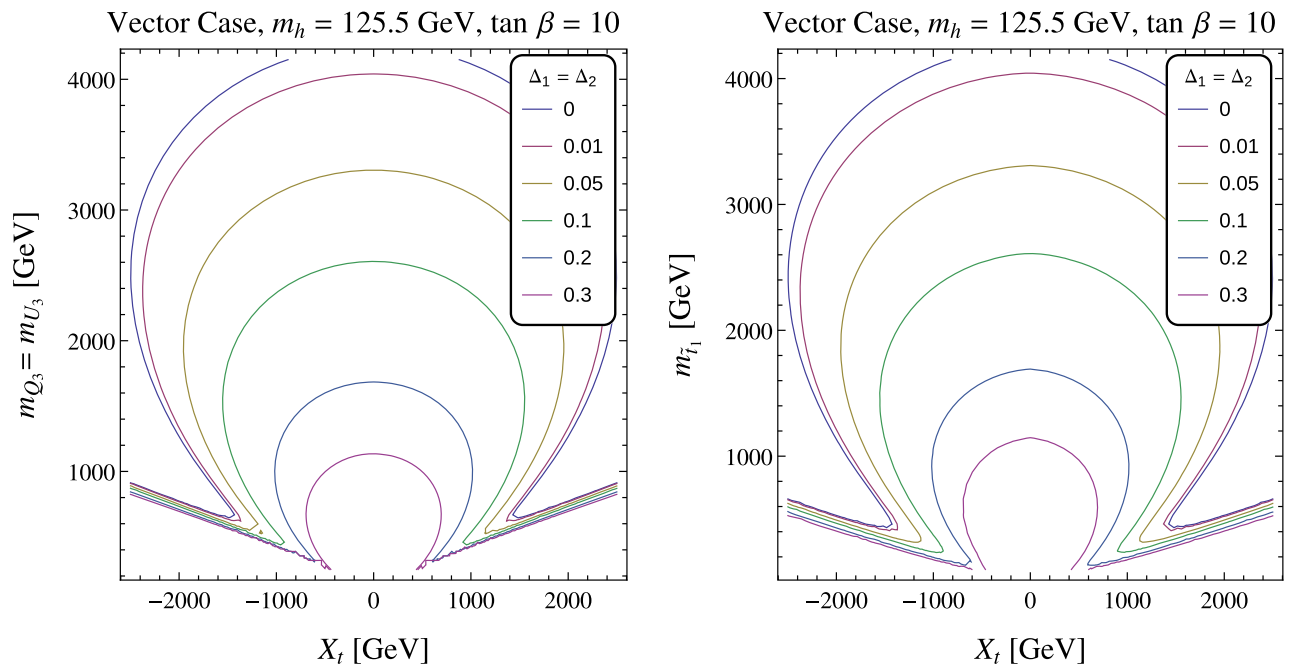

Fig. 2 Contours of the Higgs mass $m_{h}=125.5 \mathrm{GeV}$ in the $\left(m_{Q_{3}}, X_{t}\right)$ plane (left panel) and in the $\left(m_{\tilde{t}_{1}}, X_{t}\right)$ plane (right panel) for different values of $\Delta_{1}=\Delta_{2}$. We set $m_{Q_{3}}=m_{U_{3}}, \tan \beta=10$. The one-loop Higgs mass with tree-level $D$-terms corrections $m_{h, 1}$ is plotted
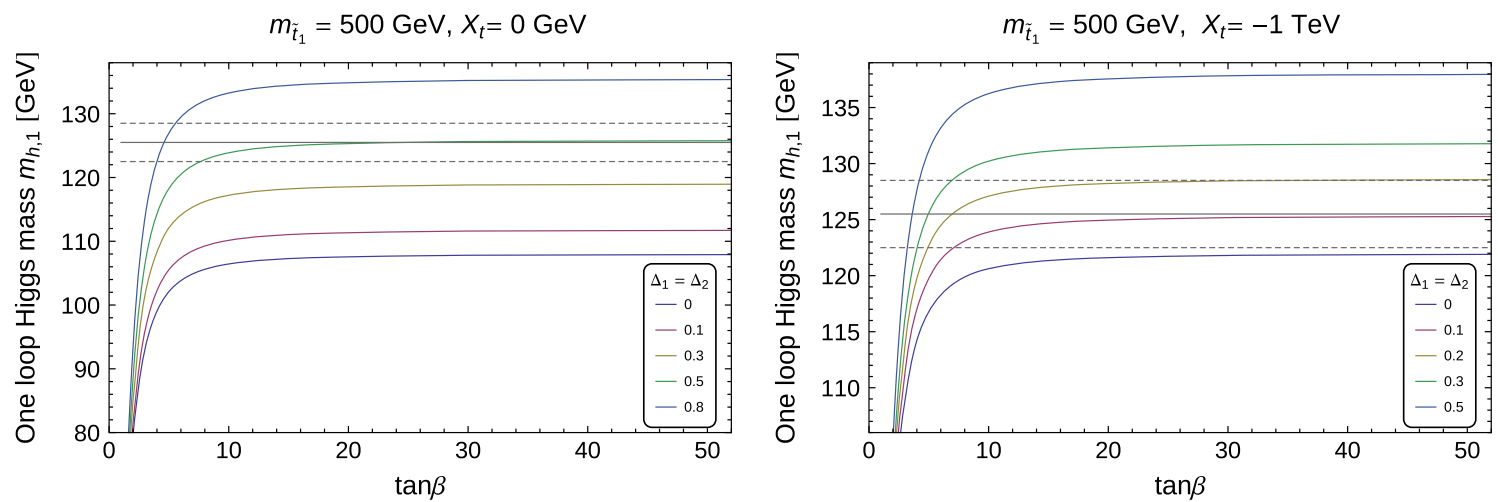

Fig. 3 One-loop Higgs mass $m_{h, 1}$ with tree-level $D$-terms corrections vs. $\tan \beta$ for different values of $\Delta_{1}=\Delta_{2}$ with $X_{t}=0$ (left panel) and $X_{t}=-1 \mathrm{TeV}$ (right panel), and with $m_{\tilde{t}_{1}}=500 \mathrm{GeV}$. For comparison, $125.5 \pm 3 \mathrm{GeV}$ grid lines are plotted

and preferable if one is to accommodate perturbative unification (see also Sect. 2.2.3). As these $\mathcal{O}(0.1) \Delta$ can still have a noticeable effect on the Higgs mass but may have a less easily observable deviation from the MSSM, we study here the degree to which their effects can be determined at the LHC and ILC.

In Figs. 3 and 4 one can see how enhancements due to the non-decoupling $D$-terms arise significantly for $\tan \beta \in$ $[1,10]$, where it is harder to reproduce $m_{h}=125.5 \mathrm{GeV}$, and stabilises for $\tan \beta \gtrsim 10$. Such results are similarly reproduced using the RG-evolution approach as in [23]. In particular in Fig. 4 it is evident that for an increasing value of $\Delta$, a lower $m_{\tilde{t}_{1}}$ is required to get $m_{h}=125.5 \mathrm{GeV}$, especially compared to the MSSM limit of $\Delta=0$.

In the left panel of Fig. 5 we can see that to have null mixing $X_{t}=0 \mathrm{GeV}$ with $\tan \beta=10, m_{\tilde{t}_{1}}$ has to be in the $1-4 \mathrm{TeV}$ range for $\Delta \in[0.01,0.3]$. On the right panel we see that for the same values of $\Delta$ with a lower stop mass $\left(m_{\tilde{t}_{1}} \sim 500 \mathrm{GeV}\right)$ still a $\left|X_{t}\right| \sim 1 \mathrm{TeV}$ is required, with negative values of $X_{t}$ preferred by theory due to RGE effects, which makes $A_{t}=X_{t}+\mu \cot \beta$ run negative. In summary, whilst the maximal mixing scenario is favoured, it is now much more achievable, due to the $D$-term effects, for smaller values of $A_{t}$, and even allows sub $2 \mathrm{TeV}$ stops for the null or small mixing scenario, when $\Delta \geq 0.3$.

The vector Higgs $D$-term extensions of the MSSM may feature different generations of matter located on different sites, for example having the first two generation matters on site $B$ [27,28], while typically the third generation is on the same site as $H_{\mathrm{u}}$ since the stop mixing parameter $X_{t}$ helps to trigger EWSB. In an alternative version of the vector-Higgs $D$-terms, the Higgses are both on site $B$. The corresponding $D$-terms are now given by Eq. (2.13) with $\Delta_{1}$ and $\Delta_{2}$, respectively, equal to 

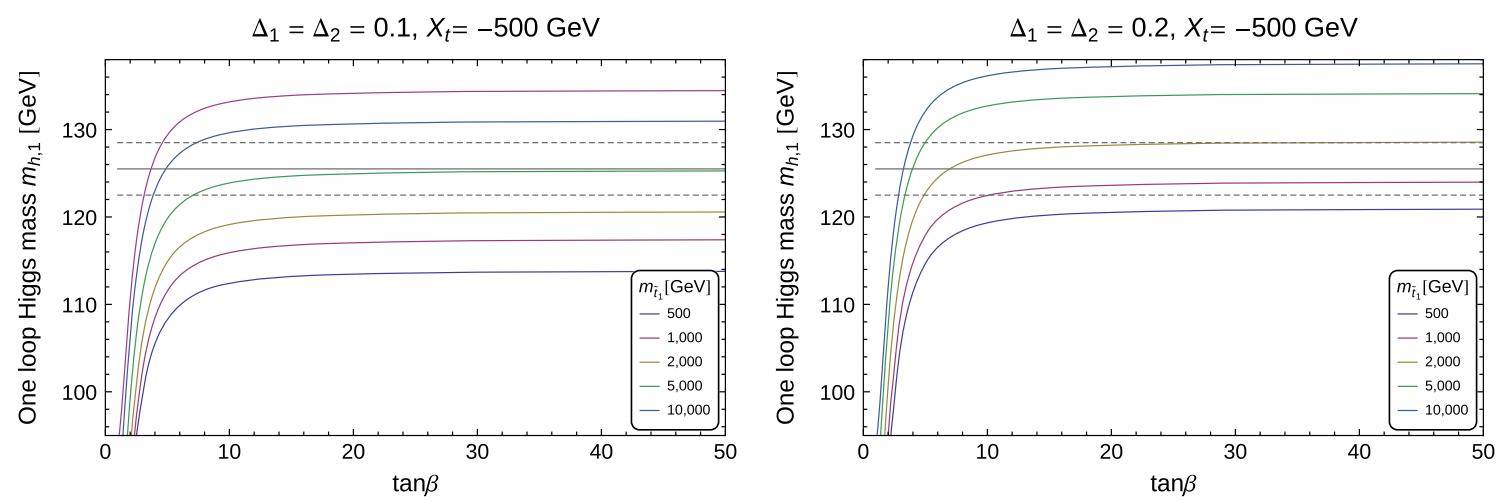

Fig. 4 One-loop Higgs mass $m_{h, 1}$ with tree-level $D$-terms corrections vs. $\tan \beta$ for different values of $m_{\tilde{t}_{1}}$ with $\Delta_{1}=\Delta_{2}=0.1$ (left panel) and $\Delta_{1}=\Delta_{2}=0.2$ (right panel), with $X_{t}=-500 \mathrm{GeV}$. For comparison, $125.5 \pm 3 \mathrm{GeV}$ grid lines are plotted
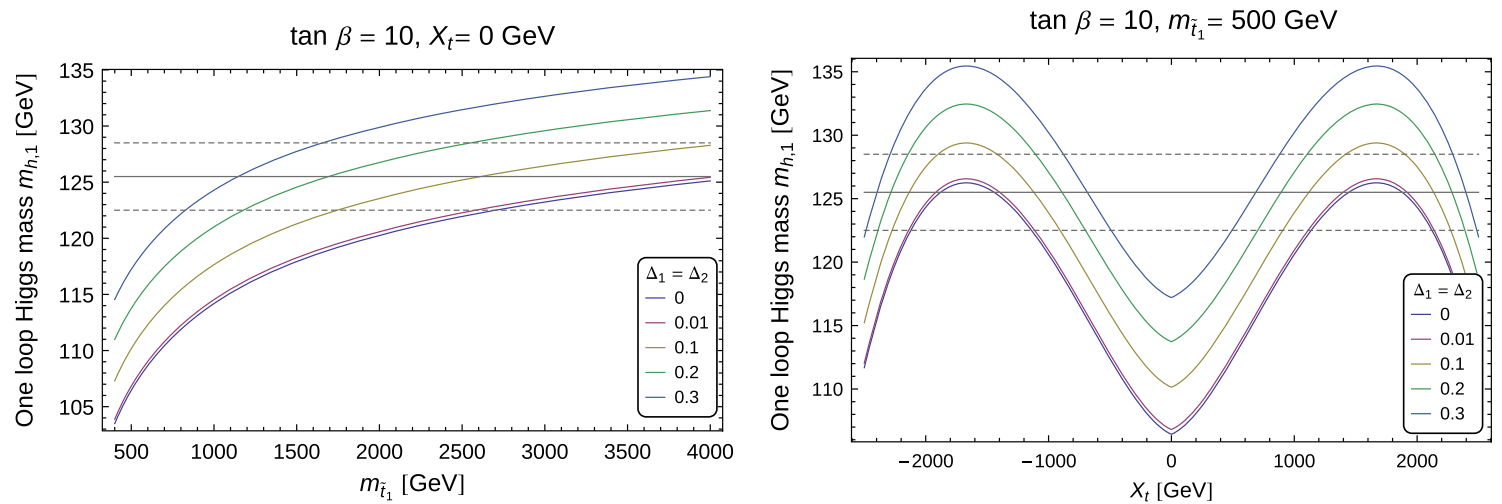

Fig. 5 One-loop Higgs mass $m_{h, 1}$ with tree-level $D$-terms corrections for different values of $\Delta_{1}=\Delta_{2}$, with $\tan \beta=10$ and $125.5 \pm 3 \mathrm{GeV}$ grid lines plotted for comparison. On the left panel, $m_{h, 1}$ vs. $m_{\tilde{t}_{1}}$ with $X_{t}=0 \mathrm{GeV}$; on the right panel, $m_{h, 1}$ vs. $X_{t}$ with $m_{\tilde{t}_{1}}=500 \mathrm{GeV}$

$\Delta_{1}^{B}=\left(\frac{g_{B 1}^{2}}{g_{A 1}^{2}}\right) \frac{m_{L}^{2}}{m_{v 1}^{2}+m_{L}^{2}}, \quad \Delta_{2}^{B}=\left(\frac{g_{B 2}^{2}}{g_{A 2}^{2}}\right) \frac{m_{L}^{2}}{m_{v 2}^{2}+m_{L}^{2}}$.

Notice that the role of the gauge couplings are reversed with respect to the model of Table 1 , having $g_{B 1}^{2}>g_{A 1}^{2}$. This can result in an easier perturbative unification if more matter is on site $A$ than site $B$ [19], although this can generate problems with EWSB. This has effects on naturalness too, depending on where the source of supersymmetry breaking is introduced, in the context of supersymmetry breaking, for instance Non-universal UV Higgs soft masses may be required to trigger EWSB at low scales.

\subsubsection{Additional fine-tuning and the Higgs mass}

We should also consider the effect on naturalness of the explicit breaking of supersymmetry from the non-decoupled $D$-terms in the EFT we restrict to. Using a cut-off, the nondecoupled $D$-terms in the vector case will lead to a quadratic divergence that contributes to the Higgs mass counterterm at one loop $[19,36]$ : $\delta m_{h, 1}^{2, \mathrm{vec}}=\left(\frac{\alpha \frac{3}{5} g_{1}^{2} \Delta_{1}+\beta g_{2}^{2} \Delta_{2}}{4}\right) \frac{M^{2}}{16 \pi^{2}}$,

where $\alpha, \beta$ are determined by the precise matter content that appears in the non-decoupling $D$-term, each generating a one-loop contribution (see Sect. A), and $M^{2}=m_{L}^{2}$. Such an effect may arise both in the Higgs tadpole equations and in the one-loop Higgs self energies. In a supersymmetric theory that is only softly broken all quadratic divergences cancel exactly at all orders in perturbation theory. In this case, Eq. (2.36) gives an additional contribution depending on the size of $M^{2}$, which should then not be too large in order not to have too much additional fine-tuning. This fine-tuning, $F$, may be quantified as

$\frac{\delta m_{h, 1}^{2, \text { vec }}}{m_{h}^{2}}=1 / F$.

In either case we have assumed in this paper, as in $[19,36]$, that $M$ is small enough (3-10 TeV) such that this contribution is neglected. It is interesting to consider the inclusion of these terms if one considers larger values of $M^{2}$ such as might arise from future $Z^{\prime}$ exclusions. 


\subsubsection{Perturbative unification and the size of the D-terms}

Here we briefly discuss gauge unification in these models as a guide to constrain the maximum possible size of $D$-terms.

To maximise the effect of the vector-like $D$-terms such as Eq. (2.14), one requires that the ratio of gauge couplings

$R_{i}=g_{A i}^{2} / g_{B i}^{2}$,

is as large as possible, however, making certain gauge couplings large at low energies and including additional matter fields will certainly effect perturbativity of the gauge couplings at higher energies. In addition, whilst these models do not (yet) have full GUT multiplets of matter, particularly for the linking fields, but also for the MSSM matter content, we can still explore the possibility of unification in these models as usual. For definiteness we take the model outlined in Table 1.

The beta functions at one loop are given by

$\beta_{g_{a}}=\frac{\mathrm{d}}{\mathrm{d} t} g_{a}=\frac{b_{a}}{16 \pi^{2}} g_{a}^{3}$ with $b_{a}=\left(2, \frac{39}{5},-5, \frac{6}{5},-3\right)$,

The restriction that $\alpha_{i}\left(M_{\mathrm{GUT}}\right)<1$ and that

$\alpha_{g_{1 A}}\left(M_{\mathrm{GUT}}\right)=\alpha_{g_{2 A}}\left(M_{\mathrm{GUT}}\right)=\alpha_{g_{3}}\left(M_{\mathrm{GUT}}\right)$,

with

$\alpha_{g_{1 B}}\left(M_{\mathrm{GUT}}\right)=\alpha_{g_{2 B}}\left(M_{\mathrm{GUT}}\right)$,

restricts the parameter space significantly. The results of perturbative unification for the largest values of $R$ 's are plotted in Fig. 6. We find $R_{1} \sim 0.6$ and $R_{2} \sim 0.86$, such that even allowing for $\frac{m_{L}^{2}}{m_{v}^{2}+m_{L}^{2}} \sim 1$ this gives

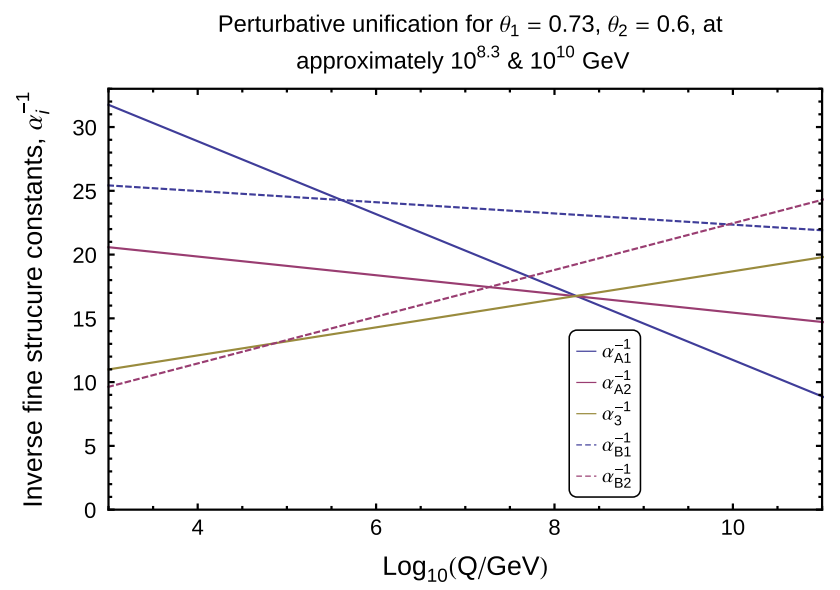

Fig. 6 Perturbative unification of the $G_{A} \otimes S U(3)_{c}$ and $G_{B}$ sites separately, allowing for the maximal value of the ratios $R_{i}$. These also give a prediction of the values of the mixing angles, $\theta_{i}$ 's in Eq. (2.15)

$$
\begin{gathered}
\Delta=\left(\frac{g_{A}^{2}}{g_{B}^{2}}\right) \frac{m_{L}^{2}}{m_{v}^{2}+m_{L}^{2}}<R \text { which implies that } \\
\left(\Delta_{1}^{\operatorname{Max}}, \Delta_{2}^{\operatorname{Max}}\right)=(0.6,0.86),
\end{gathered}
$$

respectively. Larger $\Delta$ 's are also possible if $S U$ (3) is quivered to $S U(3)_{A} \otimes S U(3)_{B}$ as then $\alpha_{3 A}^{-1}$ can be made weaker allowing for unification at a later scale and therefore larger $R$ 's. Of course abandoning perturbativity altogether will allow for a larger $D$-term enhancement too.

The dynamics of the Yukawa couplings are also of interest. The superpotential, including $K$, is given by

$W=W_{\mathrm{MSSM}}+\frac{Y_{k}}{2} K\left(L \tilde{L}-V^{2}\right)$,

in which $Y_{k}$ is taken to be real. The Yukawa coupling oneloop beta functions (for the third generation only) are given by

$$
\begin{aligned}
\beta_{y_{t}}^{(1)} & \equiv \frac{\mathrm{d}}{\mathrm{d} t} y_{t} \\
& \simeq \frac{y_{t}}{16 \pi^{2}}\left[3 y_{t}^{*} y_{t}+y_{b}^{*} y_{b}-\frac{16}{3} g_{3}^{2}-3 g_{A 2}^{2}-\frac{13}{15} g_{A 1}^{2}\right],
\end{aligned}
$$

$$
\begin{aligned}
\beta_{y_{b}}^{(1)} & \equiv \frac{\mathrm{d}}{\mathrm{d} t} y_{b} \\
& \simeq \frac{y_{b}}{16 \pi^{2}}\left[y_{t}^{*} y_{t}+y_{\tau}^{*} y_{\tau}+3 y_{b}^{*} y_{b}-\frac{16}{3} g_{3}^{2}-3 g_{A 2}^{2}-\frac{7}{15} g_{A 1}^{2}\right],
\end{aligned}
$$

$$
\begin{aligned}
\beta_{y_{\tau}}^{(1)} & \equiv \frac{\mathrm{d}}{\mathrm{d} t} y_{\tau} \\
& \simeq \frac{y_{\tau}}{16 \pi^{2}}\left[4 y_{\tau}^{*} y_{\tau}+y_{t}^{*} y_{t}-\frac{16}{3} g_{3}^{2}-3 g_{A 2}^{2}-\frac{7}{15} g_{A 1}^{2}\right],
\end{aligned}
$$

and

$$
\begin{aligned}
\beta_{y_{k}}^{(1)} & \equiv \frac{\mathrm{d}}{\mathrm{d} t} y_{k} \\
& \simeq \frac{y_{k}}{16 \pi^{2}}\left[\frac{15}{10} y_{k}^{*} y_{k}-10 g_{B 2}^{2}-10 g_{A 2}^{2}-6 g_{A 1}^{2}-6 g_{B 1}^{2}\right] .
\end{aligned}
$$

For the same choice of parameters as before, the results are presented in Fig. 7, where one can see that as $\alpha_{A 1}$ hits a Landau pole at around $10^{14} \mathrm{GeV}$, which is after both GUT scales, the Yukawa couplings become very small and run to opposite signed values. This is reminiscent of five-dimensional extensions of the MSSM $[47,48]$, where power law running is used to argue for an explanation of the order $O$ (1) top Yukawa from an initially small coupling in the UV.

\subsection{Chiral Higgs Quiver Model}

Another possible two-sites quiver is the chiral Higgs case $[21,22]$, in which the two MSSM Higgs doublets are on two 


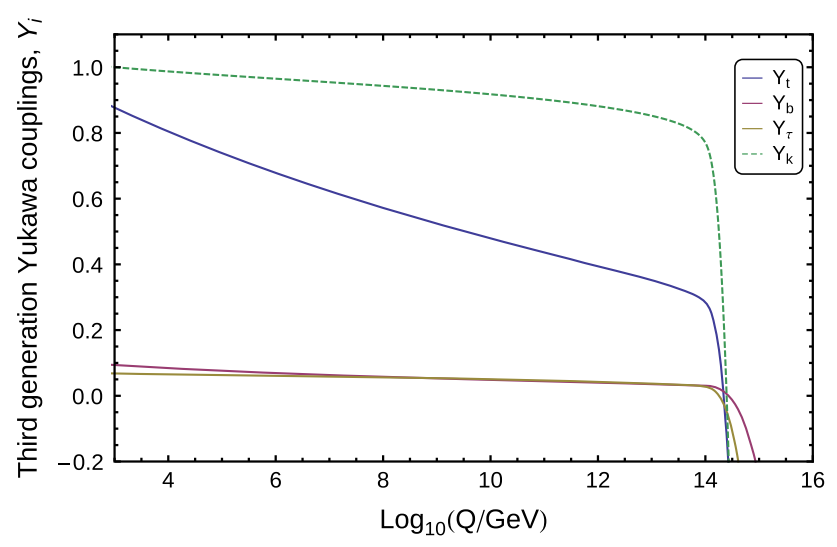

Fig. 7 Renormalisation group evolution for the Yukawa couplings

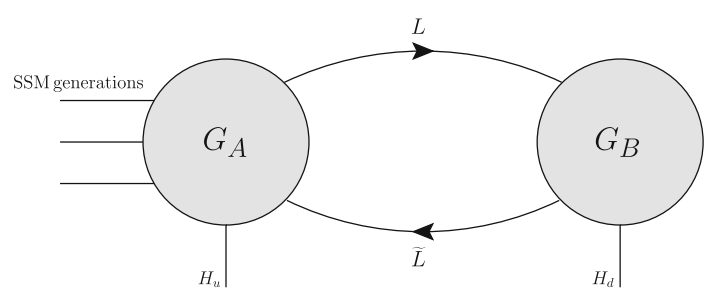

Fig. 8 The quiver module of the electroweak sector for the chiral Higgs case. The resulting chiral-Higgs-like non-decoupling $D$-term is reported in Eq. (2.48) and whose matter content is in Table 2. The model requires additional fields carrying Higgs-like charges or leptons multiplets on site $B$ instead of $A$, for anomaly cancellation

Table 2 The matter content of a quiver model that may lead to the Chiral Higgs case and the $D$-term enhancement of Eq. (2.48). This is pictured in Fig. 8. The model requires additional fields carrying Higgslike charges or leptons multiplets on site $B$ instead of $A$, for anomaly cancellation

\begin{tabular}{llll}
\hline Superfields & Spin 0 & Spin $\frac{1}{2}$ & $G_{A} \otimes G_{B} \otimes S U(3)_{c}$ \\
\hline$\hat{q}^{f}$ & $\tilde{q}^{f}$ & $q^{f}$ & $\left(\mathbf{2}, \frac{1}{6}, \mathbf{1}, 0, \mathbf{3}\right)$ \\
$\hat{d}^{f}$ & $\tilde{d}_{R}^{f *}$ & $d_{R}^{f *}$ & $\left(\mathbf{1}, \frac{1}{3}, \mathbf{1}, 0, \overline{\mathbf{3}}\right)$ \\
$\hat{u}^{f}$ & $\tilde{u}_{R}^{f *}$ & $u_{R}^{f *}$ & $\left(\mathbf{1},-\frac{2}{3}, \mathbf{1}, 0, \overline{\mathbf{3}}\right)$ \\
$\hat{l}^{f}$ & $\tilde{l}^{f}$ & $l^{f}$ & $\left(\mathbf{2},-\frac{1}{2}, \mathbf{1}, 0, \mathbf{1}\right)$ \\
$\hat{e}^{f}$ & $\tilde{e}_{R}^{f *}$ & $e_{R}^{f *}$ & $(\mathbf{1}, 1, \mathbf{1}, 0, \mathbf{1})$ \\
$\hat{H}_{\mathrm{u}}$ & $H_{\mathrm{u}}$ & $\tilde{H}_{\mathrm{u}}$ & $\left(\mathbf{2}, \frac{1}{2}, \mathbf{1}, 0, \mathbf{1}\right)$ \\
$\hat{H}_{\mathrm{d}}$ & $H_{\mathrm{d}}$ & $\tilde{H}_{\mathrm{d}}$ & $\left(\mathbf{1}, 0, \mathbf{2},-\frac{1}{2}, \mathbf{1}\right)$ \\
$\hat{L}$ & $L$ & $\psi_{L}$ & $\left(\mathbf{2},-\frac{1}{2}, \overline{\mathbf{2}}, \frac{1}{2}, \mathbf{1}\right)$ \\
$\hat{\tilde{L}}$ & $\tilde{L}$ & $\psi_{\tilde{L}}$ & $\left(\overline{\mathbf{2}}, \frac{1}{2}, \mathbf{2},-\frac{1}{2}, \mathbf{1}\right)$ \\
$\hat{K}$ & $K$ & $\psi_{K}$ & $(\mathbf{1}, 0, \mathbf{1}, 0, \mathbf{1})$ \\
\hline
\end{tabular}

alternate sites. This is pictured in Fig. 8 and in Table 2, in which the up-type Higgs doublet $H_{\mathrm{u}}$ and the three generations of matter are on site $A$, while the down-type Higgs doublet $H_{\mathrm{d}}$ is on site $B$.

The chiral Higgs case may be quite naturally achieved from a four-Higgs doublet model in which each site has two Higgs doublets and then at lower energies a Higgs doublet for each site is integrated out, resulting in a two-Higgs doublet model. In the chiral Higgs model the non-decoupling $D$ terms that are added to the scalar potential of the MSSM, at low energies, are given by

$$
\begin{aligned}
\delta \mathcal{L}= & -\frac{3}{5} \frac{g_{1}^{2} \Omega_{1}}{8}\left(\xi_{1} H_{\mathrm{u}}^{\dagger} H_{\mathrm{u}}+\frac{1}{\xi_{1}} H_{\mathrm{d}}^{\dagger} H_{\mathrm{d}}\right)^{2} \\
& -\frac{g_{2}^{2} \Omega_{2}}{8} \sum_{a}\left(\xi_{2} H_{\mathrm{u}}^{\dagger} \sigma^{a} H_{\mathrm{u}}-\frac{1}{\xi_{2}} H_{\mathrm{d}}^{\dagger} \sigma^{a} H_{\mathrm{d}}\right)^{2}+\cdots
\end{aligned}
$$

The ellipses represent terms involving other scalar particles as reported in Appendix A, while

$\xi_{i}=\frac{g_{A i}}{g_{B i}}, \quad \Omega_{1}=\frac{m_{L}^{2}}{m_{v 1}^{2}+m_{L}^{2}}, \quad \Omega_{2}=\frac{m_{L}^{2}}{m_{v 2}^{2}+m_{L}^{2}}$,

and $\Delta_{i}=\xi_{i}^{2} \cdot \Omega_{i}$, with $i=1,2$. The minimisation conditions now take the form

$$
\begin{array}{r}
m_{H_{\mathrm{u}}}^{2}+|\mu|^{2}-B_{\mu} \cot \beta-\frac{m_{Z}^{2}}{2} \cos (2 \beta)+m_{\Omega}^{2} \cos ^{2} \beta+C=0, \\
m_{H_{\mathrm{d}}}^{2}+|\mu|^{2}-B_{\mu} \tan \beta+\frac{m_{Z}^{2}}{2} \cos (2 \beta)+m_{\Omega}^{2} \sin ^{2} \beta+D=0,
\end{array}
$$

where

$m_{\Omega}^{2}=\frac{v^{2}}{8} \sum_{i=1,2} k_{i} g_{i}^{2} \Omega_{i}$

with $k_{i}=(3 / 5,1)$ and

$$
\begin{aligned}
& C=\frac{v^{2}}{8} \sum_{i=1,2} k_{i} g_{i}^{2} \Omega_{i} \xi_{i}^{2} \sin ^{2} \beta, \\
& D=\frac{v^{2}}{8} \sum_{i=1,2} k_{i} g_{i}^{2} \Omega_{i} \frac{\cos ^{2} \beta}{\xi_{i}^{2}} .
\end{aligned}
$$

Equations (2.50), (2.51) then give

$$
\begin{aligned}
& \sin (2 \beta)=\frac{2 B_{\mu}}{m_{H_{\mathrm{u}}}^{2}+m_{H_{\mathrm{d}}}^{2}+2|\mu|^{2}+C+D+m_{\Omega}^{2}}, \\
& m_{Z}^{2}=\frac{2}{1-\tan ^{2} \beta}\left[\left(C+m_{H_{\mathrm{u}}}^{2}\right) \tan ^{2} \beta\right. \\
& \left.-\left(D+m_{H_{\mathrm{d}}}^{2}\right)\right]-2|\mu|^{2} .
\end{aligned}
$$

The following Higgs mass matrices may be derived. In the basis of the real components of $\left(H_{\mathrm{d}}^{0}, H_{\mathrm{u}}^{0}\right)$, the mass matrix for the CP-even Higgses given by

$$
\left.\begin{array}{l}
m_{h}^{2}= \\
\left(\begin{array}{c}
m_{h, 11} \\
\frac{v_{\mathrm{d}} v_{\mathrm{u}}}{4} \sum_{i} k_{i} g_{i}^{2}\left(\Omega_{i}-1\right)-\operatorname{Re}\left[B_{\mu}\right]
\end{array} \frac{v_{\mathrm{d}} v_{\mathrm{u}}}{4} \sum_{i} k_{i} g_{i}^{2}\left(\Omega_{i}-1\right)-\operatorname{Re}\left[B_{\mu}\right]\right. \\
m_{h, 22}
\end{array}\right),
$$



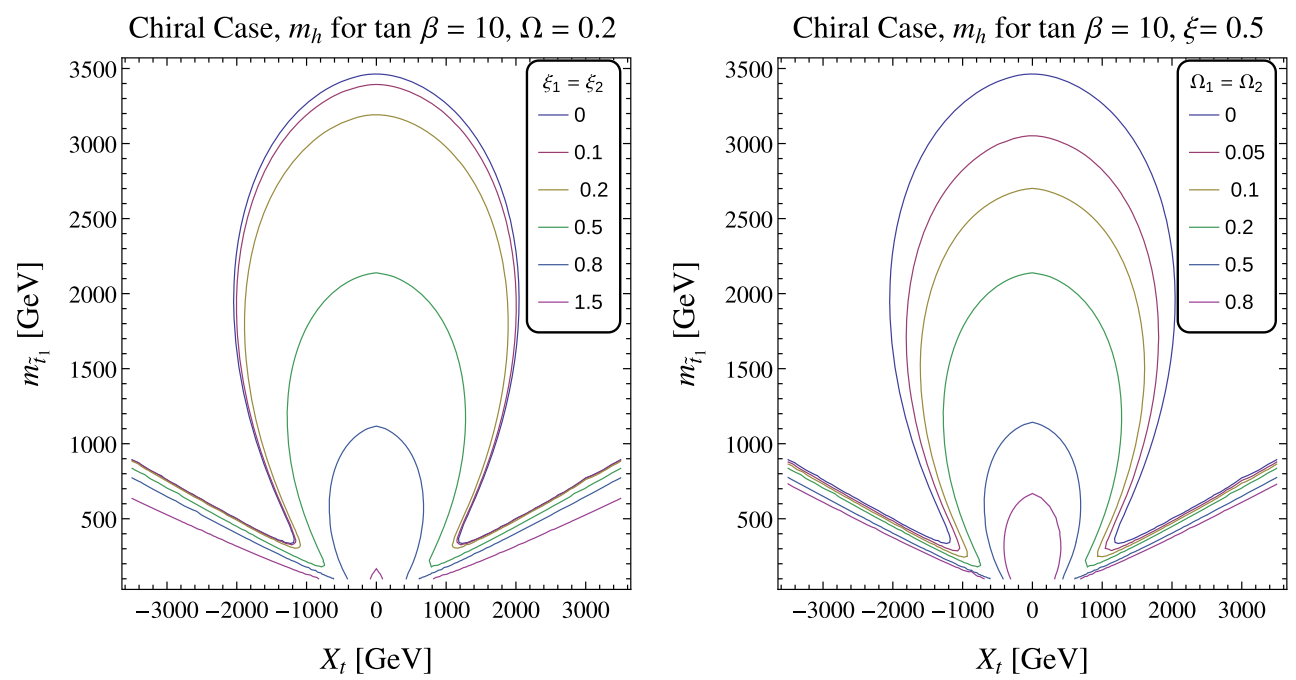

Fig. 9 Contours of the Higgs mass $m_{h}=125.5 \mathrm{GeV}$ in the $\left(m_{\tilde{t}_{1}}, X_{t}\right)$ plane for different values of $\xi$ (left panel) and $\Omega$ (right panel), with $m_{Q_{3}}=m_{U_{3}}, \tan \beta=10$. The one-loop Higgs mass with tree-level $D$-terms corrections $m_{h, 1}$ is plotted

with

$m_{h, 11}=B_{\mu} \tan \beta+m_{Z}^{2} \cos ^{2} \beta+\frac{v^{2}}{4} \cos ^{2} \beta \sum_{i} k_{i} g_{i}^{2} \frac{\Omega_{i}}{\xi_{i}^{2}}$,

$m_{h, 22}=B_{\mu} \cot \beta+m_{Z}^{2} \sin ^{2} \beta+\frac{v^{2}}{4} \sin ^{2} \beta \sum_{i} k_{i} g_{i}^{2} \Omega_{i} \xi_{i}^{2}$.

For the pseudo-scalar Higgses, the mass matrix in the basis of the imaginary components of $\left(H_{\mathrm{d}}^{0}, H_{\mathrm{u}}^{0}\right)$ reads

$m_{A^{0}}^{2}=\left(\begin{array}{ll}m_{A^{0}, 11} & \operatorname{Re}\left[B_{\mu}\right] \\ \operatorname{Re}\left[B_{\mu}\right] & m_{A^{0}, 22}\end{array}\right)$,

with

$m_{A^{0}, 11}=B_{\mu} \cot \beta, \quad m_{A^{0}, 22}=B_{\mu} \tan \beta$.

The mass matrix for charged Higgses $\left(H_{\mathrm{d}}^{-}, H_{\mathrm{u}}^{+, *}\right)$, $\left(H_{\mathrm{d}}^{-, *}, H_{\mathrm{u}}^{+}\right)$is

$m_{H^{-}}^{2}=\left(\begin{array}{cc}m_{H^{-}, 11} & \frac{1}{4} g_{2}^{2}\left(1-\Omega_{2}\right) v_{\mathrm{d}} v_{\mathrm{u}}+B_{\mu}^{*} \\ \frac{1}{4} g_{2}^{2}\left(1-\Omega_{2}\right) v_{\mathrm{d}} v_{\mathrm{u}}+B_{\mu} & m_{H^{-}, 22}\end{array}\right)$,

with

$m_{H^{-}, 11}=B_{\mu} \tan \beta+m_{\mathrm{W}}^{2} \sin ^{2} \beta\left(1-\Omega_{2}\right)$,

$m_{H^{-}, 22}=B_{\mu} \cot \beta+m_{\mathrm{W}}^{2} \cos ^{2} \beta\left(1-\Omega_{2}\right)$.

The masses of the Higgs states are adjusted accordingly $m_{h^{0}, H^{0}}^{2, \operatorname{chir}}=\frac{1}{2}\left(m_{A}^{2}+m_{Z}^{2}\right)+(C+D)$

$\mp \frac{1}{2} \sqrt{\left(m_{A}^{2}-m_{Z}^{2}+\frac{2(C-D)}{\cos (2 \beta)}\right)^{2} c^{2}(2 \beta)+\left(m_{A}^{2}+m_{Z}^{2}-2 m_{\Omega}^{2}\right)^{2} s^{2}(2 \beta)}$,

$m_{A}^{2, \text { chir }} \equiv \frac{2 B_{\mu}}{\sin 2 \beta}=m_{H_{\mathrm{u}}}^{2}+m_{H_{\mathrm{d}}}^{2}+2|\mu|^{2}+C+D+m_{\Omega}^{2}$,

$m_{H^{ \pm}}^{2, \text { chir }}=m_{A}^{2}+m_{\mathrm{W}}^{2}\left(1-\Omega_{2}\right)$,

where $c^{2}(2 \beta)=\cos ^{2}(2 \beta), s^{2}(2 \beta)=\sin ^{2}(2 \beta)$. The nondecoupling $D$-terms in this model lead to a shift to the treelevel mass, which in the leading order in the $1 / \tan \beta$ expansion is given by

$m_{h, 0}^{2} \simeq\left[m_{Z}^{2}+\left(\frac{\frac{3}{5} g_{1}^{2} \xi_{1}^{2} \Omega_{1}+g_{2}^{2} \xi_{2}^{2} \Omega_{2}}{4}\right) v^{2}\right]+\mathcal{O}\left(\frac{1}{\tan ^{2} \beta}, \xi_{i}\right)$.

\subsubsection{Higgs mass enhancement in the chiral Higgs case}

We discuss the impact of non-decoupling $D$-terms in the chiral Higgs case on the Higgs mass, taking for simplicity $\Omega \equiv \Omega_{1}=\Omega_{2}$ and $\xi \equiv \xi_{1}=\xi_{2}$. In Fig. 9 is plotted the Higgs mass from Eq. (2.12) with the tree-level $D$-term corrections from (2.67) in the $\left(m_{\tilde{t}_{1}}, X_{t}\right)$ plane for different values of $\xi$ or $\Omega$. Also in this case the $125.5 \mathrm{GeV}$ contour lines show that the $D$-term contribution lower the minimal stop masses required for a given value of $X_{t}$. In Fig. 10, in a fashion similar to [22], ${ }^{5}$ we show the $m_{h}$ contour lines in the $(\xi, \Omega)$-plane for $m_{\tilde{t}_{1}}=500 \mathrm{GeV}$ and $1 \mathrm{TeV}$.

In the chiral Higgs case, too, the explicit supersymmetry breaking in the low energy effective theory leads to a reasoning similar to the one discussed in Sect. 2.2.2.

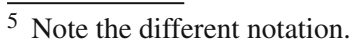



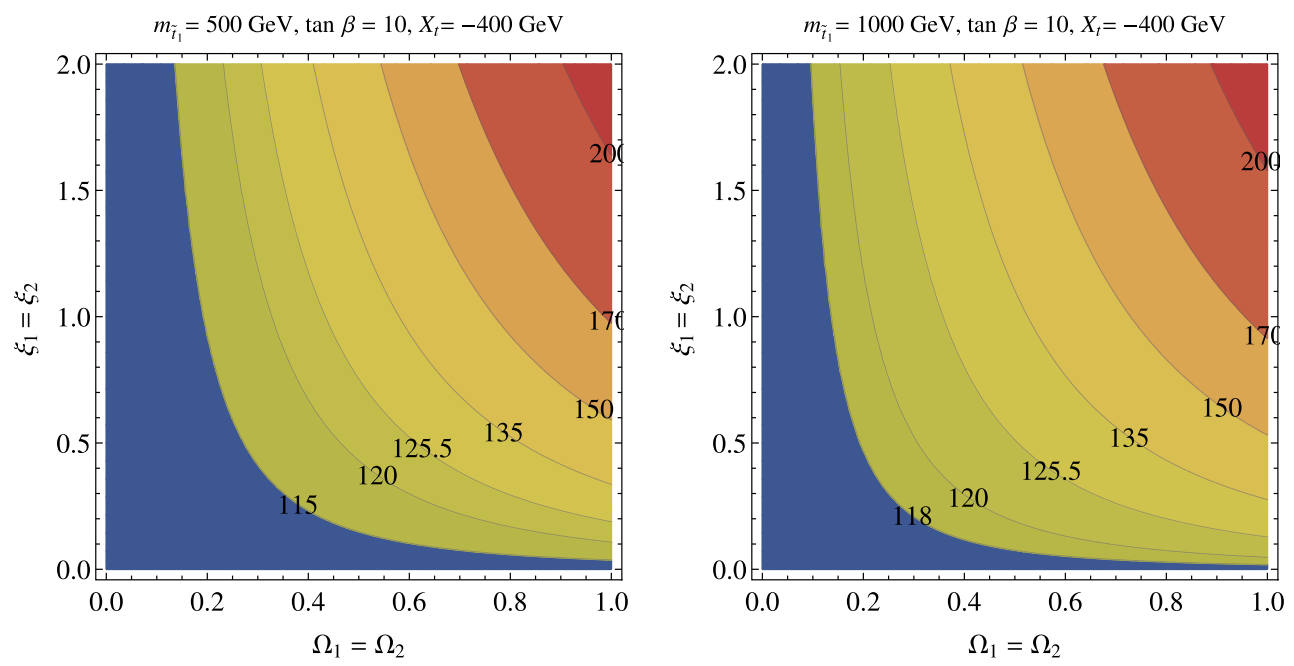

Fig. 10 The Higgs mass in the $\left(\xi_{1}=\xi_{2}, \Omega_{1}=\Omega_{2}\right)$ plane, for the chiral Higgs case, with $m_{\tilde{t}_{1}}=500 \mathrm{GeV}$ (left panel) and $m_{\tilde{t}_{1}}=1 \mathrm{TeV}$ (right panel), while $\tan \beta=10, A_{t}=-400 \mathrm{GeV}$. The one-loop Higgs mass with tree-level $D$-terms corrections $m_{h, 1}$ is plotted

\section{Higgs couplings determination at the LHC and ILC}

Non-decoupling $D$-terms induced by the quiver extensions of the MSSM, apart from shifting the tree-level masses of the scalars of the theory (see Appendix A), have direct impact also on several physical quantities as, for instance, the $h \rightarrow$ $\gamma \gamma$ decay branching ratio [30] or the Higgs boson couplings to fermions and gauge bosons [22,36,49-51]. We will study the latter effects, estimating the dependence of the deviations from the SM couplings on the additional $D$-terms, in the light of the precise determination of Higgs boson couplings at current and future colliders. Let us then first define the ratio of the Higgs (the lightest eigenstate $h$ ) coupling normalised by that of the Standard Model couplings:

$\kappa_{U}=g_{U} / g_{U}^{\mathrm{SM}}, \quad \kappa_{D}=g_{D} / g_{D}^{\mathrm{SM}}, \quad \kappa_{V}=g_{V} / g_{V}^{\mathrm{SM}}$,

for any up(down)-type fermion $U=u, c, t(D=d, s, b, e$, $\mu, \tau$ ), or gauge boson $V=W^{ \pm}, Z$.

A standard way to express these ratios, or scaling factors, in a 2HDM models of type-II such as the MSSM, is to write them in terms of the angles $\beta$ and $\alpha$,

$\kappa_{D} \equiv-\frac{\sin \alpha}{\cos \beta}, \quad \kappa_{U} \equiv \frac{\cos \alpha}{\sin \beta}, \quad \kappa_{V} \equiv \sin (\beta-\alpha)$,

where $\alpha$ is defined as the mixing angle of the Higgs mass eigenstates,

$$
\left(\begin{array}{l}
h_{0} \\
H_{0}
\end{array}\right)=\sqrt{2}\left(\begin{array}{rr}
-\sin \alpha & \cos \alpha \\
\cos \alpha & \sin \alpha
\end{array}\right)\left(\begin{array}{l}
\operatorname{Re} H_{\mathrm{d}}^{0} \\
\operatorname{Re} H_{\mathrm{u}}^{0}
\end{array}\right) .
$$

The SM is recovered for $\sin \alpha=-\cos \beta, \cos \alpha=+\sin \beta$. We can express $\kappa_{t}, \kappa_{V}$ in terms of $\tan \beta$ and $\kappa_{b}$ (not considering wrong mixings $\Delta_{b}$ coming from loop effects) [36]:

$$
\begin{aligned}
\kappa_{t} & =\sqrt{1-\frac{\kappa_{b}^{2}-1}{\tan ^{2} \beta}} \\
\kappa_{V} & =\frac{\tan \beta}{1+\tan ^{2} \beta}\left(\frac{\kappa_{b}}{\tan \beta}+\sqrt{1+\tan ^{2} \beta-\kappa_{b}^{2}}\right) .
\end{aligned}
$$

The relations (3.2) are exact; however, a more transparent general expression for the scaling factors can be obtained looking at the specific model considered. We study models in the decoupling limit for large $\tan \beta$. A procedure to rewrite the Higgs couplings in this regime is to start from the general 2HDM Higgs scalar potential, Eq. (2.1) and integrate out the heavier states identified to the Higgs doublet $H_{\mathrm{d}}$; see also $[22,36,50]$. The Higgs couplings can be read from the effective Lagrangian after having integrated out $H_{\mathrm{d}}^{0}$ and, after a perturbative expansion in $1 / \tan \beta, \kappa_{b}=\kappa_{\tau}$ are

$\kappa_{b} \simeq\left(1-\frac{m_{h}^{2}}{m_{H}^{2}}\right)^{-1}\left(1-\frac{\left[\lambda_{3}+\lambda_{5}\right] v^{2}}{m_{H}^{2}-m_{h}^{2}}\right)+\cdots$,

where we adopt the definitions from Eq. (2.1) and the ellipses denote non-zero $\lambda_{7}$ contributions from $F$-term like enhancements that are null in the MSSM, $\mathcal{O}\left(1 / \tan ^{2} \beta\right)$ corrections and possible "wrong sign" couplings coming from 1-loop contributions. Finding the right $\kappa_{b}$ expressions for our quiver models is straightforward, substituting into (3.5) the corresponding $\lambda_{3}, \lambda_{5}$. For the vector Higgs case $\lambda_{3}, \lambda_{5}$ are obtained by the MSSM relations (2.2) with the additional contributions (2.13), giving $\lambda_{3}+\lambda_{5}=-\left[g_{2}^{2}\left(1+\Delta_{2}\right)+\right.$ $\left.\frac{3}{5} g_{1}^{2}\left(1+\Delta_{1}\right)\right] / 4$, such that

$\kappa_{b}^{\text {vector }} \simeq\left(1-\frac{m_{h}^{2}}{m_{H}^{2}}\right)^{-1}\left(1+\frac{\left[g_{2}^{2}\left(1+\Delta_{2}\right)+\frac{3}{5} g_{1}^{2}\left(1+\Delta_{1}\right)\right] v^{2}}{4\left(m_{H}^{2}-m_{h}^{2}\right)}\right)$. 


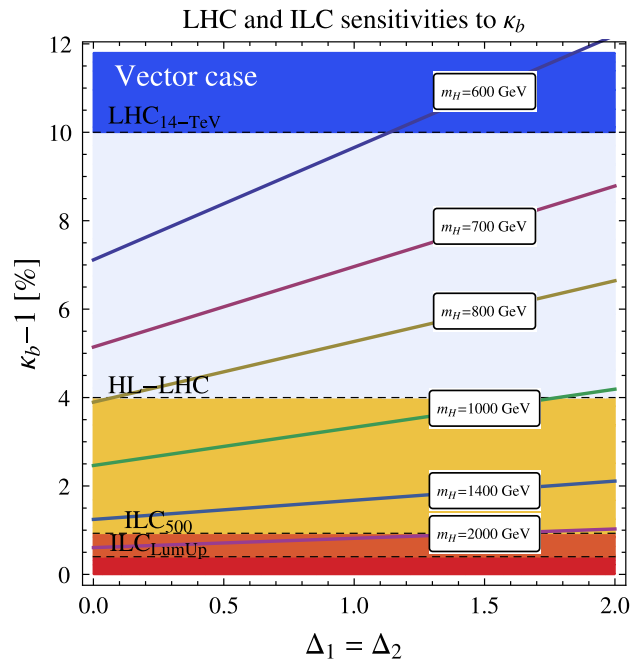

(a)

Fig. 11 Vector case: relative enhancements $\kappa_{b}-1$ of the Higgs bottom couplings for the chiral-Higgs case with respect to the SM are displayed in solid lines, in (\%) as a function of $\Delta_{1}=\Delta_{2}$, for different values of $m_{H}(\mathrm{GeV})$. a In dashed lines, the contours of the expected accuracies on the scaling factors $\kappa_{b}$ at the LHC, HL-LHC and ILC, from [52] and

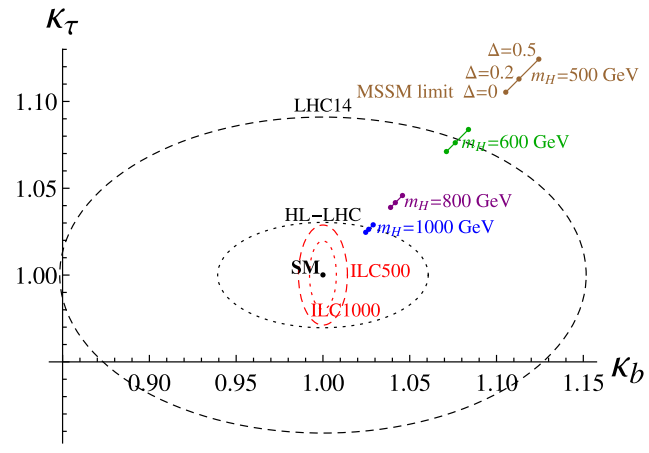

(a)

Fig. 12 Vector-Higgs case: experimental sensitivity to coupling deviations from the Standard Model, assuming no correlation between $\kappa_{i}$ measures. a $\left(\kappa_{b}, \kappa_{\tau}\right)$ for $\Delta=0$ (SM limit), 0.1, 0.2, 0.5, at different values of $m_{H}=500,600,800,1000,1200 \mathrm{GeV}$. The experimental sensitivity, centred in the SM value $\left(\kappa_{b}, \kappa_{\tau}\right)=1$, is represented by $1 \sigma$ -

For the chiral Higgs case, using instead the additional contributions (2.48), one obtains $\lambda_{3}+\lambda_{5}=-\left[g_{2}^{2}\left(1-\Omega_{2}\right)+\right.$ $\left.\frac{3}{5} g_{1}^{2}\left(1-\Omega_{1}\right)\right] / 4$, and

$\kappa_{b}^{\text {chiral }} \simeq\left(1-\frac{m_{h}^{2}}{m_{H}^{2}}\right)^{-1}\left(1+\frac{\left[g_{2}^{2}\left(1-\Omega_{2}\right)+\frac{3}{5} g_{1}^{2}\left(1-\Omega_{1}\right)\right] v^{2}}{4\left(m_{H}^{2}-m_{h}^{2}\right)}\right)$.

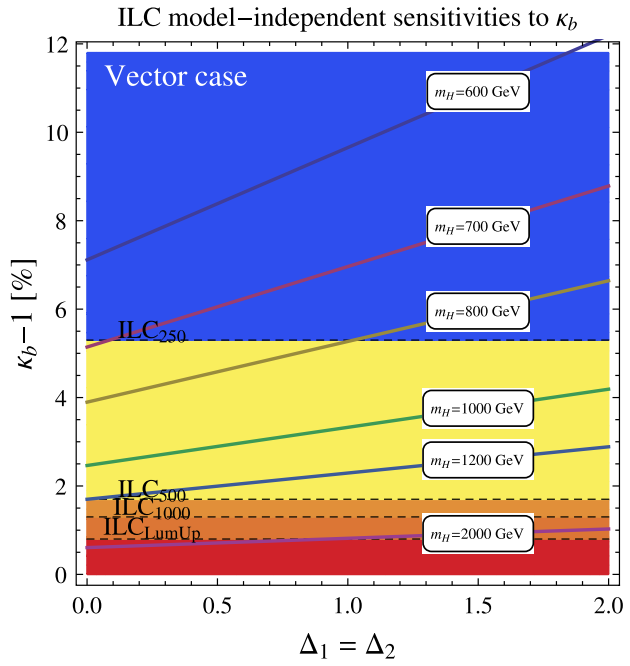

(b)

Table 3, centred on the SM value 0 . The accuracies assume no non-SM production and decay modes and assumes universality $\left(\kappa_{\mathrm{u}} \equiv \kappa_{t}=\kappa_{c}\right.$, $\kappa_{\mathrm{d}} \equiv \kappa_{b}=\kappa_{s}$ and $\left.\kappa_{l} \equiv \kappa_{\tau}=\kappa_{\mu}\right)$. b In dashed lines, the contours of the model-independent ILC sensitivities for each run from [53]; see Table 4, centred on the SM value 0

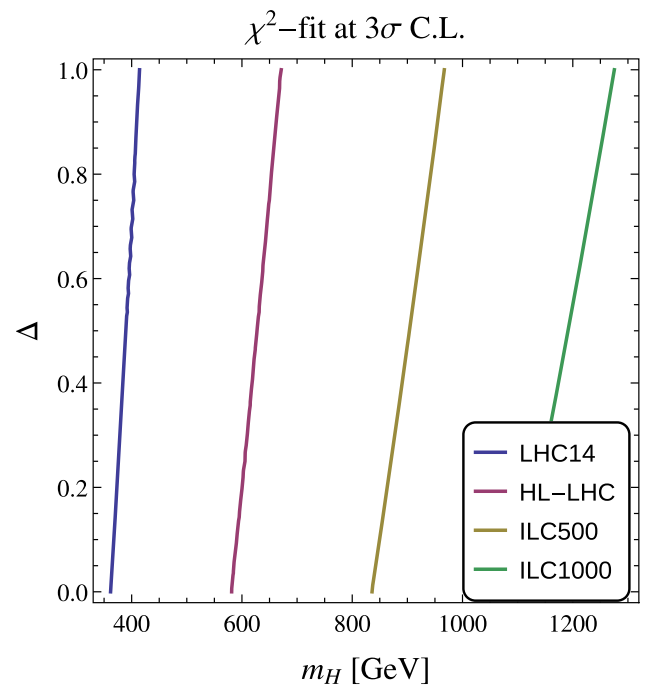

(b)

confidence ellipses: black dashed for $\mathrm{LHC}$ at $14 \mathrm{TeV}$ and $300 \mathrm{fb}^{-1}$, black dotted for HL-LHC at $3000 \mathrm{fb}^{-1}$ at $14 \mathrm{TeV}$, red dashed ILC at $500 \mathrm{GeV}$ and red dotted for ILC at $1000 \mathrm{GeV} . \mathbf{b} \chi^{2}$-test of $\kappa_{\mathrm{W}}, \kappa_{Z}, \kappa_{\tau}, \kappa_{b}, \kappa_{t}$ in the $\left(m_{H}, \Delta\right)$-plane at the different experiments: areas on the left of the solid lines are not consistent with the SM at $3 \sigma$-confidence level

In these two cases, the MSSM limit can be obtained by setting the non-decoupling $D$-term contributions to zero, respectively $\Delta_{i}=0$ and $\Omega_{i}=0$.

It is important to understand how these $D$-term enhanced deviations from the SM couplings could be detected, as a signature for the considered quiver models at present and future colliders; see Figs. 11, 12, 13, 14. At the LHC only ratios 


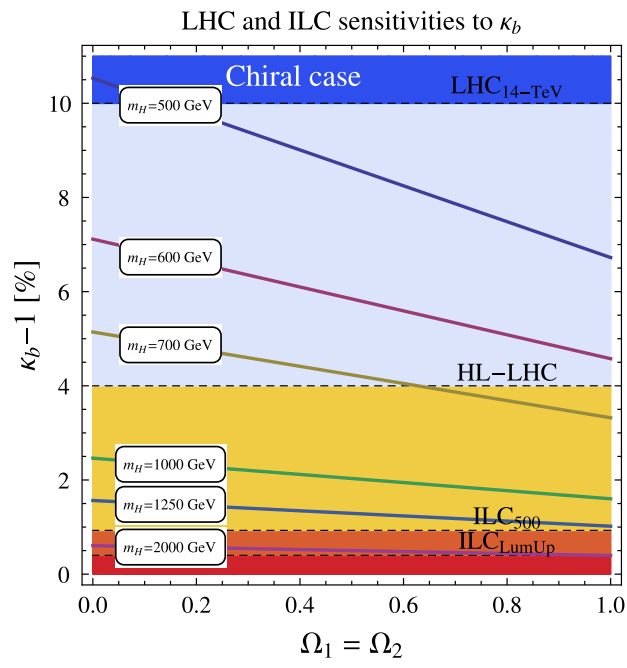

(a)

Fig. 13 Chiral-Higgs case: relative enhancements $\kappa_{b}-1$ of the Higgs bottom couplings for the chiral-Higgs case with respect to the SM are displayed in solid lines, in (\%) as a function of $\Omega_{1}=\Omega_{2}$ for different values of $m_{H}(\mathrm{GeV})$. a In dashed lines, the contours of the expected accuracies on the scaling factors $\kappa_{b}$ at the LHC, HL-LHC and ILC, from [52] and Table 3, centred on the SM value 0 . The accuracies

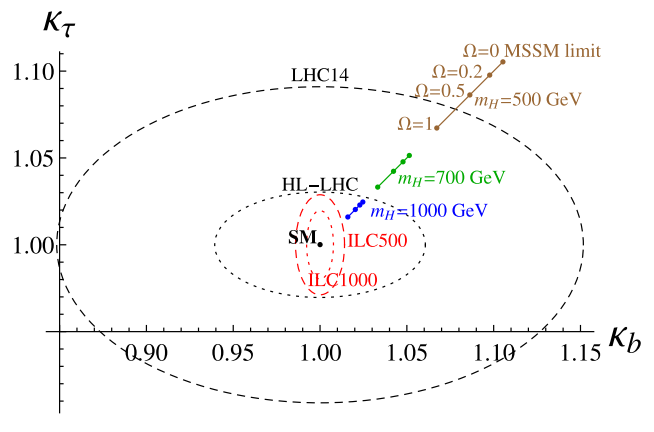

(a)

Fig. 14 Chiral-Higgs case: experimental sensitivity to coupling deviations from the Standard Model, assuming no correlation between $\kappa_{i}$ measures. a $\left(\kappa_{b}, \kappa_{\tau}\right)$ for $\Omega=0$ (SM limit), 0.2, 0.5, 1 at different values of $m_{H}=500,600,800,1000,1200 \mathrm{GeV}$. The experimental sensitivity, centred in the SM value $\left(\kappa_{b}, \kappa_{\tau}\right)=1$, is represented by $1 \sigma$ -

between different Higgs couplings can be determined, therefore coupling determination is possible only in the framework of a specific model. For example, taking some minimal assumptions on the underlying model, as explained in [52], one can obtain $\kappa_{b}$ from a constrained 7-parameter fit assuming no non-SM production and decay modes and assuming

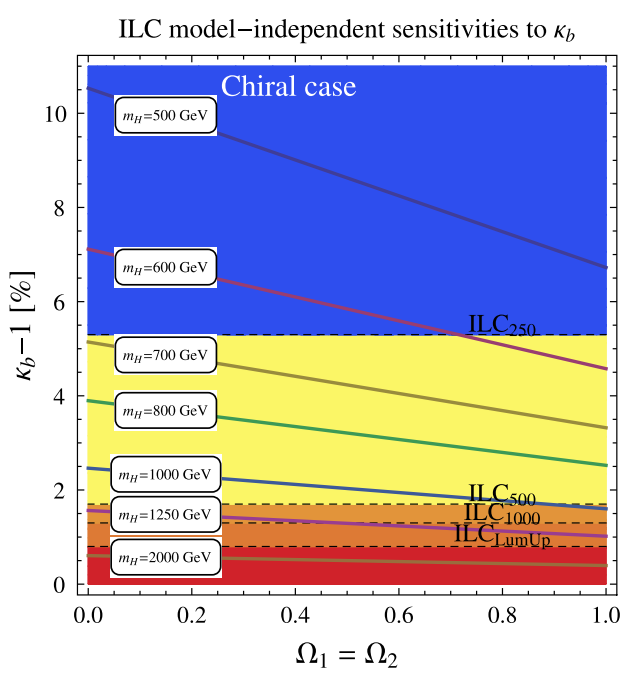

(b)

assume no non-SM production and decay modes and assumes universality $\left(\kappa_{\mathrm{u}} \equiv \kappa_{t}=\kappa_{c}, \kappa_{\mathrm{d}} \equiv \kappa_{b}=\kappa_{s}\right.$ and $\left.\kappa_{l} \equiv \kappa_{\tau}=\kappa_{\mu}\right)$. Correlations are neglected. $\mathbf{b}$ In dashed lines, the contours of the model-independent ILC sensitivities for each run from [53], see Table 4, centred on the SM value 0

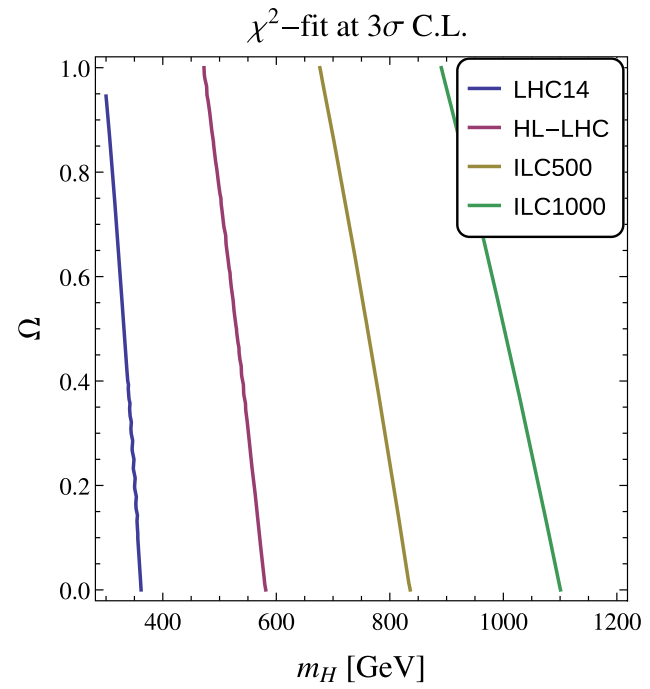

(b)

confidence ellipses: black dashed for LHC at $14 \mathrm{TeV}$ and $300 \mathrm{fb}^{-1}$, black dotted for HL-LHC at $3000 \mathrm{fb}^{-1}$ at $14 \mathrm{TeV}$, red dashed ILC at $500 \mathrm{GeV}$ and red dotted for ILC at $1000 \mathrm{GeV} . \mathbf{b} \chi^{2}$-test of $\kappa_{\mathrm{W}}, \kappa_{Z}, \kappa_{\tau}, \kappa_{b}, \kappa_{t}$ in the $\left(m_{H}, \Delta\right)$-plane at the different experiments: areas on the left of the solid lines are not consistent with the SM at $3 \sigma$-confidence level

generation universality $\left(\kappa_{\mathrm{u}} \equiv \kappa_{t}=\kappa_{c}, \kappa_{\mathrm{d}} \equiv \kappa_{b}=\kappa_{s}\right.$ and $\kappa_{l} \equiv \kappa_{\tau}=\kappa_{\mu}$ ). This is listed in Table 3, where the coupling determination uncertainties at $\mathrm{LHC}$ at $14 \mathrm{TeV}$ $\left(\int \mathcal{L} \mathrm{d} t=300 \mathrm{fb}^{-1}\right)$ and High Luminosity LHC (HL-LHC, $\left.\int \mathcal{L} \mathrm{d} t=3000 \mathrm{fb}^{-1}\right)$ are compared with some expectations at the International Linear Collider (ILC). 
Table 3 Expected precisions on $\kappa_{b}$ at $1 \sigma$, in $\%$, from a constrained 7-parameter fit assuming no non-SM production and decay modes and assuming universality $\left(\kappa_{\mathrm{u}} \equiv \kappa_{t}=\kappa_{c}, \kappa_{\mathrm{d}} \equiv \kappa_{b}=\kappa_{s}\right.$ and $\kappa_{l} \equiv \kappa_{\tau}=\kappa_{\mu}$ ), as reported in [52]. LHC corresponds to $300 \mathrm{fb}^{-1}$ at $14 \mathrm{TeV}, \mathrm{HL}-\mathrm{LHC}$ at $3000 \mathrm{fb}^{-1}$ at $14 \mathrm{TeV}$

\begin{tabular}{lllllll}
\hline & LHC 14 $(\%)$ & HL-LHC $(\%)$ & $\operatorname{ILC}_{500}(\%)$ & $\operatorname{ILC}_{500-\text { LumUp }}(\%)$ & ILC $_{1000}(\%)$ & ILC $_{1000-L_{u m U p}}(\%)$ \\
\hline$\kappa_{\mathrm{W}}$ & $4-6$ & $2-5$ & 0.39 & 0.21 & 0.21 & 0.2 \\
$\kappa_{Z}$ & $4-6$ & $2-4$ & 0.49 & 0.24 & 0.5 & 0.3 \\
$\kappa_{l}=\kappa_{\tau}$ & $6-8$ & $2-5$ & 1.9 & 0.98 & 1.3 & 0.72 \\
$\kappa_{\mathrm{d}}=\kappa_{b}$ & $10-13$ & $4-7$ & 0.93 & 0.60 & 0.51 & 0.4 \\
$\kappa_{\mathrm{u}}=\kappa_{t}$ & $14-15$ & $7-10$ & 2.5 & 1.3 & 1.3 & 0.9 \\
\hline
\end{tabular}

Table 4 Expected accuracies on the coupling scaling factors $\kappa_{i}$ at $1 \sigma$, in $\%$, for a completely model-independent fit assuming theory errors $\Delta F_{i} / F_{i}=0.5 \%$, from the ILC Higgs White Paper [53]

\begin{tabular}{lllll}
\hline & $\operatorname{ILC}_{250}(\%)$ & $\operatorname{ILC}_{500}(\%)$ & $\operatorname{ILC}_{1000}(\%)$ & $\operatorname{ILC}_{\text {LumUp }}(\%)$ \\
\hline$\kappa_{\mathrm{W}}$ & 4.9 & 1.2 & 1.1 & 0.6 \\
$\kappa_{Z}$ & 1.3 & 1.0 & 1.0 & 0.5 \\
$\kappa_{\tau}$ & 5.8 & 2.4 & 1.8 & 1.0 \\
$\kappa_{b}$ & 5.3 & 1.7 & 1.3 & 0.8 \\
$\kappa_{t}$ & - & 14 & 3.2 & 2.0 \\
\hline
\end{tabular}

On the other hand, at future $e^{+} e^{-}$-colliders as the ILC, the Higgs total width and the Higgs couplings can be determined in a model-independent way. This is possible by exploiting the recoil methods that allow for a decay independent determination of the Higgsstrahlung process production $e^{+} e^{-} \rightarrow H Z$, a quantity that enters many observables [53]. With respect to estimates with minimal model assumption, there are slightly higher uncertainties. This is reported in Table 4, where we show the estimated ILC accuracies on the Higgs couplings, assuming the theoretical uncertainties to be equal to $0.5 \%$ for the ILC stages at $\sqrt{s}=250,500,1000$ $\mathrm{GeV}$ and for the luminosity upgrade ILC $_{\text {LumUp }}$ at 250, 500, $1000 \mathrm{GeV}$, from [53], which may be further improved [54]. Since ILC measurements are dominated by statistical errors, they are improved with increasing statistics, in contrast with Higgs determinations in the High-Luminosity LHC that are dominated by systematic errors.

In Fig. 11 we plot how the LHC and ILC may detect deviations from the SM Higgs bottom coupling due to nondecoupling $D$-terms in a vector Higgs quiver extension of the MSSM. The relative enhancement with respect to the SM Higgs bottom coupling, $\kappa_{b}-1$, is plotted as a function of $\Delta$ for different values of the heavier neutral CP-even Higgs mass $m_{H}$ (see Eq. (3.6)). The non-decoupling $D$-terms in the vector Higgs case enhance the deviation from the SM with respect to the MSSM limit $\Delta=0$, while larger values for $m_{H}$ clearly suppress these effects. Furthermore, in Fig. $11 \mathrm{a}$ value of $\kappa_{b}-1$ that lies above a contour line corresponds to a deviation from the SM that can be detected, once the Higgs bottom coupling at the corresponding machine run is mea- sured. In Fig. 11a, the horizontal dashed contour lines correspond to the LHC and ILC $1 \sigma$-confidence level sensitivities for $\kappa_{b}$ determination with the minimal model assumptions in Table 3 , centred on the SM value $\kappa_{b}^{S M}-1=0$. In Fig. 11b, the sensitivities for ILC model-independent $\kappa_{b}$ determination are displayed. At the $\mathrm{LHC}$ at $14 \mathrm{TeV}$, deviations triggered by $\Delta \sim \mathcal{O}(1-2)$ may be detected for a $m_{H} \lesssim 600 \mathrm{GeV}$, while for $m_{H} \leq 1 \mathrm{TeV}$, these deviations may be detected at the HLLHC. Coupling enhancements due to $\Delta \sim \mathcal{O}(0.1-0.2)$, are more suitable according to the top-down approach in [23], are (just) discernible at the HL-LHC for $m_{H} \lesssim 800 \mathrm{GeV}$. The ILC running at $500 \mathrm{GeV}$ may be sensitive to these ranges of $\Delta$ for $m_{H} \sim 1 \mathrm{TeV}$, while for the high luminosity configuration at $1000 \mathrm{GeV}\left(\int \mathcal{L} \mathrm{d} t=\mathcal{O}(5000) \mathrm{fb}^{-1}\right)$, this is valid up to $m_{H} \sim 2 \mathrm{TeV}$, showing the power of this experiment in the study of the Higgs scalar potential.

The deviation from the SM of $\kappa_{b}$ in Fig. 11 alone cannot be used for claiming a BSM underlying model, as it might merely be due to statistical effects. Therefore, in Fig. 12a we show the non-decoupling $D$-terms triggered deviations in $\kappa_{b}$ and $\kappa_{\tau}$ : the points lying outside the $1 \sigma$-confidence ellipses for each experiment can be distinguished from the SM. ${ }^{6}$ In Fig. 12b we perform a $\chi^{2}$-fit to the SM values of $\kappa_{\mathrm{W}}, \kappa_{Z}, \kappa_{\tau}, \kappa_{b}, \kappa_{t}$ in the $\left(m_{H}, \Delta\right)$-plane: the areas on the left of the solid lines are not consistent with the SM at $3 \sigma$-confidence level. As deviations from the SM value 1 for $\kappa_{\mathrm{W}}, \kappa_{Z}, \kappa_{t}$ are relatively mild in these models, see Eq. (3.4), in particular considering the achievable accuracy in these quantities, the main contribution to the $\chi^{2}$ result comes from $\kappa_{b}$ and $\kappa_{\tau}$, as they present large deviations and a relatively good resolution. One can see that at the first run of the LHC deviations from the Standard Model only for a relatively light $H$, with mass up to $m_{H} \simeq 350-400 \mathrm{GeV}$ are observable and the luminosity upgrade is needed to explore the parameter space up to decoupling masses $m_{H} \lesssim 500 \mathrm{GeV}$ for any value of $\Delta$. At the ILC, instead, deviations from the SM for $m_{H}$ up to $700(900) \mathrm{GeV}$ at $\sqrt{s}=500(1000) \mathrm{GeV}$. In both

\footnotetext{
6 A similar kind of analysis, for general 2HDM models, may be found in [55].
} 
plots in Fig. 12 we do not take into account any experimental correlations between the determinations of $\kappa_{i}$.

In the chiral Higgs quiver case, the $D$-terms triggered deviations of $\kappa_{b}$ (in particular), have an opposite behaviour compared to the vector case. Here, the $D$-term contributions are negative, see Eq. (3.7), pushing the Higgs couplings closer to the SM value. Therefore for increasing $\Omega$, the deviations of the couplings from the SM get less detectable with respect to the MSSM limit (for $\Omega=0$ ); see Figs. 13 and 14a. Figure 14b shows how, for $\Omega \sim \mathcal{O}(1)$, the sensitivity to the deviation of couplings is reduced at the LHC by $\sim 50 \mathrm{GeV}$ and by $\sim 100 \mathrm{GeV}$ at the ILC.

Once a deviation in the couplings from the SM is detected, one should address the question of which (BSM) supersymmetric model has been observed. For this, the measurement of the couplings alone is not sufficient, but also the detection of $H$ and the measurement of its mass $m_{H}$ are fundamental, as one can decouple the $\Delta$ or $\Omega$ measurement; see Eq. (3.6).

\section{Conclusions}

In this paper we explored two cases in which non-decoupled $D$-terms arise. We studied how the size of this enhancement affects the light $\mathcal{C P}$-even Higgs mass and its couplings to fermions and vector bosons in the decoupling limit $m_{A} \gg$ $m_{Z}$ with large $\tan \beta$. We found that:

- In the vector Higgs case, a $D$-term size parameter $\Delta$ of order $\sim \mathcal{O}$ (1) allows one to reach a Higgs mass of 125.5 $\mathrm{GeV}$ at tree level, with consequent relaxation of naturalness. On the other hand, $\Delta \sim \mathcal{O}(0.1)$ may still provide a suitable enhancement to the Higgs mass, and seem to be preferred in the light of perturbative unification, from a top down approach. Similar conclusions can be drawn in the chiral Higgs case.

- The Higgs couplings to $b$ and $\tau$ are particularly sensitive to deviations from SM and MSSM values due to nondecoupling $D$-terms, and can be studied at the LHC and ILC. For a more decoupled heavy Higgs $H$, i.e. larger $m_{H}$, the deviations from the SM couplings are smaller. In the vector Higgs case these deviations increase for larger $D$ terms, and can be determined at the HL-LHC for any value of $\Delta$ with $m_{H} \lesssim 600 \mathrm{GeV}$. At the ILC at $500 \mathrm{GeV}$ the sensitivity to deviations is much improved, and deviations from the SM for $0 \leq \Delta \leq 0.5$ can be seen with $m_{H} \leq$ 800-900 GeV; further improvement is possible with the ILC 1-TeV upgrade.

- In the chiral Higgs case, instead, contributions from the non-decoupling $D$-terms reduce the deviations in the couplings from the SM value and result in a more challenging experimental determination. At the ILC at $500 \mathrm{TeV}$, in correspondence of a maximal $D$-terms contribution to the Higgs mass $(\Omega=1)$, the sensitivity is reduced, the deviations from the SM being detectable with $m_{H} \leq 650$ $\mathrm{GeV}$.

Once deviations from SM couplings are established, in order to distinguish the model from the MSSM, a precise measurement of $m_{H}$ is required to obtain the $D$-terms parameters. Furthermore, combining these results with electroweak precision measurements where the effects of gauge extensions could be observed, may possibly identify these models. In order to be sensitive to a vast range of gauge extended models, we have shown that the precise and largely modelindependent measurements of the Higgs couplings at the linear collider is needed.

Acknowledgments The authors would like to thank K. Blum and R.T. D'Agnolo for comments regarding [50]. M.M. would like to thank A. Bharucha and A. Goudelis. S.P. would like to thank P. Drechsel, K. Fujii, S. Kanemura, F. Moortgat, G. Nardini, M. Tonini, M. de Vries and G. Weiglein and acknowledges the support of DFG through the grant SFB 676 "Particles, Strings, and the Early Universe". This work was partially supported by the Foundation for Polish Science International $\mathrm{PhD}$ Projects Programme co-financed by the EU European Regional Development Fund. This work has been partially supported by National Science Centre under research grant DEC-2012/04/A/ST2/00099.

Open Access This article is distributed under the terms of the Creative Commons Attribution License which permits any use, distribution, and reproduction in any medium, provided the original author(s) and the source are credited.

Funded by $\mathrm{SCOAP}^{3}$ / License Version CC BY 4.0.

\section{Appendix A: General derivation of non-decoupling D-terms}

Here we give a derivation of the non-decoupling $D$-terms and show how they may arise within a two-site quiver model, involving scalars charged under the final symmetry: squarks, sleptons as well as Higgs bosons. We consider the product of two identical (non-)abelian gauge groups $G_{A} \otimes G_{B}$ that breaks to the diagonal subgroup, $G_{D}$. The canonical kinetic terms for $i$ chiral superfields $A_{i}$, charged under only $G_{A}$, and of $j$ chiral superfields $B_{j}$, charged under only $G_{B}$, are given by

$\mathcal{L} \supset \int \mathrm{d}^{4} \theta\left(\sum_{i} A_{i}^{\dagger} \mathrm{e}^{g_{a} V_{a}} A_{i}+\sum_{j} B_{j}^{\dagger} \mathrm{e}^{g_{b} V_{b}} B_{j}\right)$,

where $g_{a}$ and $g_{b}$, respectively, are the gauge couplings for site $A$ and $B$ and $V_{a}, V_{b}$ are the corresponding vector multiplets.

After the diagonal breaking $G_{A} \otimes G_{B} \rightarrow G_{D}, V_{a}$ and $V_{b}$ recombine into a massless vector multiplet, $V_{D}$, and a heavy one, $V_{H}$, which can be written as 
$V_{D}=\frac{g_{a} V_{b}+g_{b} V_{a}}{\sqrt{g_{a}^{2}+g_{b}^{2}}}, \quad V_{H}=\frac{-g_{a} V_{a}+g_{b} V_{b}}{\sqrt{g_{a}^{2}+g_{b}^{2}}}$.

$V_{H}$ obtains a mass through the supersymmetric Higgs mechanism by eating a (complex) chiral superfield $\Phi$, in our case a combination of the linking fields between the sites $A$ and $B$,

$\Phi=(t+i s)+\sqrt{2} \theta \chi+\theta^{2} F_{\Phi}$.

The real scalar field $t$ is eaten to give the third degree of freedom to the gauge fields $A_{\mu}, s$ remains uneaten, while the Weyl fermion $\chi$ couples to the gaugino $\lambda$ to make a supersymmetric Dirac mass. In the Kähler potential the corresponding mass term $m_{V}^{2}$ for $V_{H}$ is given by

$\mathcal{L} \supset \int \mathrm{d}^{4} \theta m_{V}^{2} V_{H}^{2}+\cdots$

Furthermore, the following soft mass terms are added:

$$
\begin{aligned}
\mathcal{L} & \supset \int \mathrm{d}^{4} \theta\left(m_{\chi} m_{V}^{2} \theta^{2}+\bar{m}_{\chi} m_{V}^{2} \bar{\theta}^{2}-\frac{1}{2} m_{V}^{2} m_{s}^{2} \theta^{4}\right) V_{H}^{2} \\
& +\int \mathrm{d}^{2} \theta m_{\lambda} W_{\alpha}^{2}+\int \mathrm{d}^{2} \bar{\theta} \bar{m}_{\lambda} \bar{W}_{\dot{\alpha}}^{2}
\end{aligned}
$$

where the soft masses $m_{\chi}, m_{s}^{2}, m_{\lambda}$, respectively, parametrise the soft breaking of the fermion $\chi$, the real uneaten scalar $s$ and the usual Majorana soft mass for the gaugino $\lambda$. Therefore the Kähler potential may be written to leading order in $V_{H}$ as

$$
K_{H} \supset g_{\mathrm{d}}\left(\frac{g_{a}}{g_{b}}\right) \mathcal{J}_{a} V_{H}+g_{\mathrm{d}}\left(\frac{g_{b}}{g_{a}}\right) \mathcal{J}_{b} V_{H}+\cdots .
$$

$\mathcal{J}_{a / b}$ are the current multiplets, satisfying the constraint $D^{2} \mathcal{J}=0$, that contain all the fields charged under site $A$ or site $B$ :

$$
\begin{aligned}
\mathcal{J}^{c}= & J^{c}+i \theta j^{c}-i \bar{\theta} \bar{j}^{c}-\theta \sigma^{\mu} \bar{\theta} j_{\mu}^{c}+\frac{1}{2} \theta \theta \bar{\theta} \bar{\sigma}^{\mu} \partial_{\mu} j^{c} \\
& -\frac{1}{2} \bar{\theta} \bar{\theta} \theta \sigma^{\mu} \partial_{\mu} \bar{j}^{c}-\frac{1}{4} \theta \theta \bar{\theta} \bar{\theta} \square J^{c},
\end{aligned}
$$

with the leading term being the current of scalars $J^{c}=$ $\sum_{i} \phi_{i}^{\dagger} T^{c} \phi_{i}$, where $\phi_{i}$ are the collection of all scalars charged under the gauge group and $c$ is the generator index. The effective lagrangian after integrating out the heavy vector field $V_{H}$ is then of the form

$\mathcal{L}_{\text {eff }}=\int \mathrm{d}^{4} \theta\left(\sum_{i} A_{i}^{\dagger} \mathrm{e}^{g_{D} V_{D}} A_{i}+\sum_{j} B_{j}^{\dagger} \mathrm{e}^{g_{D} V_{D}} B_{j}\right)+\mathcal{O}$.

$\mathcal{O}$ is the most general expression for the non-decoupled $D$ terms,

$$
\begin{aligned}
\mathcal{O}=g_{D}^{2} & \int \mathrm{d}^{4} \theta\left(\frac{1}{m_{V}^{2}}-\frac{m_{s}^{2} \theta^{4}}{m_{V}^{2}+m_{s}^{2}}\right) \\
& \times \sum_{A}\left[\left(\frac{g_{a}}{g_{b}}\right) \mathcal{J}_{a}^{A}-\left(\frac{g_{b}}{g_{a}}\right) \mathcal{J}_{b}^{A}\right]^{2},
\end{aligned}
$$

with a sum over $A$ generators. The associated non-decoupling $D$-term corresponds then to the $\theta^{4}$ term in the round brackets of Eq. (A.9), while the currents in the square brackets reduce simply to $\left(\frac{1}{8}\right)$ the scalar current for this $\theta^{4}$ term.

Passing explicitly to the case of quiver extensions of the MSSM, the diagonal gauge group coupling $g_{D}$ corresponds to the SM coupling $g_{\mathrm{SM}}$ and the symmetry breaking consists in

$S U(2)_{A} \otimes S U(2)_{B} \rightarrow S U(2)_{L}, \quad U(1)_{A} \otimes U(1)_{B} \rightarrow U(1)_{Y}$.

In the case of a model in which all MSSM fields are on site $A$, charged under $G_{A}$ the scalar currents are given by

$$
\begin{aligned}
J_{U(1)_{A}}= & \frac{1}{2} H_{\mathrm{u}}^{\dagger} H_{\mathrm{u}}-\frac{1}{2} H_{\mathrm{d}}^{\dagger} H_{\mathrm{d}}-\frac{1}{2} \tilde{l}^{\dagger} \tilde{l}+\frac{1}{6} \tilde{q}^{\dagger} \tilde{q} \\
& +\frac{1}{3} \tilde{d}^{\dagger} \tilde{d}-\frac{2}{3} \tilde{u}^{\dagger} \tilde{u}+\tilde{e}^{\dagger} \tilde{e}, \\
J_{U(1)_{B}}= & 0, \\
J_{S U(2)_{A}}^{A}= & \frac{1}{2}\left(H_{\mathrm{u}}^{\dagger} \sigma^{A} H_{\mathrm{u}}+H_{\mathrm{d}}^{\dagger} \sigma^{A} H_{\mathrm{d}}+\tilde{q}^{\dagger} \sigma^{A} \tilde{q}+\tilde{l}^{\dagger} \sigma^{A} \tilde{l}\right), \\
J_{S U(2)_{B}}^{A}= & 0,
\end{aligned}
$$

with all flavour and colour indices implicitly traced. For the case of split generations (see for instance [27]), in which the third generation and $H_{\mathrm{u}}, H_{\mathrm{d}}$ are charged under $G_{A}$ and the first two generations under $G_{B}$, one finds

$$
\begin{aligned}
J_{U(1)_{A}}= & \frac{1}{2} H_{\mathrm{u}}^{\dagger} H_{\mathrm{u}}-\frac{1}{2} H_{\mathrm{d}}^{\dagger} H_{\mathrm{d}} \\
& +\left[-\frac{1}{2} \tilde{l}^{\dagger} \tilde{l}+\frac{1}{6} \tilde{q}^{\dagger} \tilde{q}+\frac{1}{3} \tilde{d}^{\dagger} \tilde{d}-\frac{2}{3} \tilde{u}^{\dagger} \tilde{u}+\tilde{e}^{\dagger} \tilde{e}\right]_{3}, \\
J_{U(1)_{B}}= & {\left[-\frac{1}{2} \tilde{l}^{\dagger} \tilde{l}+\frac{1}{6} \tilde{q}^{\dagger} \tilde{q}+\frac{1}{3} \tilde{d}^{\dagger} \tilde{d}-\frac{2}{3} \tilde{u}^{\dagger} \tilde{u}+\tilde{e}^{\dagger} \tilde{e}\right]_{1,2}, }
\end{aligned}
$$

$J_{S U(2)_{A}}^{A}=\frac{1}{2}\left(H_{\mathrm{u}}^{\dagger} \sigma^{A} H_{\mathrm{u}}+H_{\mathrm{d}}^{\dagger} \sigma^{A} H_{\mathrm{d}}\right)+\frac{1}{2}\left[\tilde{q}^{\dagger} \sigma^{A} \tilde{q}+\tilde{l}^{\dagger} \sigma^{A} \tilde{l}\right]_{3}$,

$J_{S U(2)_{B}}^{A}=+\frac{1}{2}\left[\tilde{q}^{\dagger} \sigma^{A} \tilde{q}+\tilde{l}^{\dagger} \sigma^{A} \tilde{l}\right]_{1,2}$.

These results may be extended to a four-Higgs doublet model or to a quiver model with three or more sites, straightforwardly. The sum in (A.9) implies that the $D$-terms generate mass shifts to the Higgs doublets, to all charged squarks and sleptons as well as additional quartic vertices. As a consequence, additional contributions to branching ratios should be considered in precision studies with Higgs and sfermion decays. An accurate detection of these effects may allow for 
the determination of the gauge structure and the charges of matter fields to identify the underlying model.

\section{References}

1. ATLAS Collaboration, G. Aad et al., Observation of a new particle in the search for the Standard Model Higgs boson with the ATLAS detector at the LHC. Phys. Lett. B 716, 1-29 (2012). arXiv: 1207.7214

2. CMS Collaboration, S. Chatrchyan et al., Observation of a new boson at a mass of $125 \mathrm{GeV}$ with the CMS experiment at the LHC. Phys. Lett. B 716, 30-61 (2012). arXiv:1207.7235

3. J.F. Gunion, H.E. Haber, The CP conserving two Higgs doublet model: the approach to the decoupling limit. Phys. Rev. D 67, 075019 (2003). arXiv:hep-ph/0207010

4. P. Fayet, Supergauge invariant extension of the Higgs mechanism and a model for the electron and its neutrino. Nucl. Phys. B 90, 104-124 (1975)

5. H.P. Nilles, M. Srednicki, D. Wyler, Weak interaction breakdown induced by supergravity. Phys. Lett. B 120, 346 (1983)

6. J. Frere, D. Jones, S. Raby, Fermion masses and induction of the weak scale by supergravity. Nucl. Phys. B 222, 11 (1983)

7. J. Derendinger, C.A. Savoy, Quantum effects and SU(2) x U(1) breaking in supergravity gauge theories. Nucl. Phys. B 237, 307 (1984)

8. L. Durand, J.L. Lopez, Upper bounds on Higgs and top quark masses in the flipped SU(5) x U(1) superstring model. Phys. Lett. B 217, 463 (1989)

9. J.R. Ellis, J. Gunion, H.E. Haber, L. Roszkowski, F. Zwirner, Higgs bosons in a nonminimal supersymmetric model. Phys. Rev. D 39, 844 (1989)

10. M. Maniatis, The next-to-minimal supersymmetric extension of the standard model reviewed. Int. J. Mod. Phys. A 25, 3505-3602 (2010). arXiv:0906.0777

11. U. Ellwanger, C. Hugonie, A.M. Teixeira, The next-to-minimal supersymmetric standard model. Phys. Rep. 496, 1-77 (2010). arXiv:0910.1785

12. M. Drees, R. Godbole, P. Roy, Theory and Phenomenology of Sparticles: An Account of Four-Dimensional N = 1 Supersymmetry in High Energy Physics. (World Scientific, 2004)

13. J. Espinosa, M. Quiros, Higgs triplets in the supersymmetric standard model. Nucl. Phys. B 384, 113-146 (1992)

14. J. Espinosa, M. Quiros, Upper bounds on the lightest Higgs boson mass in general supersymmetric Standard Models. Phys. Lett. B 302, 51-58 (1993). arXiv:hep-ph/9212305

15. J.R. Espinosa, M. Quiros, Gauge unification and the supersymmetric light Higgs mass. Phys. Rev. Lett. 81, 516-519 (1998). arXiv:hep-ph/9804235

16. A. Delgado, G. Nardini, M. Quiros, A light supersymmetric Higgs sector hidden by a standard model-like Higgs. JHEP 1307, 054 (2013). arXiv: 1303.0800

17. P. Bandyopadhyay, S. Di Chiara, K. Huitu, A.S. Keçeli, Naturality vs perturbativity, $\mathrm{B}_{s}$ physics, and LHC data in triplet extension of MSSM. JHEP 1411, 062 (2014). arXiv: 1407.4836

18. P. Batra, A. Delgado, D.E. Kaplan, T.M. Tait, The Higgs mass bound in gauge extensions of the minimal supersymmetric standard model. JHEP 0402, 043 (2004). arXiv:hep-ph/0309149

19. A. Maloney, A. Pierce, J.G. Wacker, D-terms, unification, and the Higgs mass. JHEP 0606, 034 (2006). arXiv:hep-ph/0409127

20. Y. Zhang, H. An, X.-D. Ji, R.N. Mohapatra, Light Higgs mass bound in SUSY left-right models. Phys. Rev. D 78, 011302 (2008). arXiv:0804.0268
21. L. Randall, Warped geometries and branes. The 10th International Conference on Supersymmetry and Unification of Fundamental Interactions SUSY'02, Hamburg, Germany, 17-23 June 2002

22. N. Craig, A. Katz, A supersymmetric Higgs sector with chiral Dterms. JHEP 1305, 015 (2013). arXiv: 1212.2635

23. A. Bharucha, A. Goudelis, M. McGarrie, En-gauging naturalness. Eur. Phys. J. C 74, 2858 (2014). arXiv: 1310.4500

24. F. Staub, SARAH, SARAH Hepforge (2008). arXiv:0806.0538

25. C. Csaki, J. Erlich, C. Grojean, G.D. Kribs, 4-D constructions of supersymmetric extra dimensions and gaugino mediation. Phys. Rev. D 65, 015003 (2002). arXiv:hep-ph/0106044

26. H. Cheng, D. Kaplan, M. Schmaltz, W. Skiba, Deconstructing gaugino mediation. Phys. Lett. B 515, 395-399 (2001). arXiv:hep-ph/0106098

27. P. Batra, A. Delgado, D.E. Kaplan, T.M. Tait, Running into new territory in SUSY parameter space. JHEP 0406, 032 (2004). arXiv:hep-ph/0404251

28. A. Delgado, Raising the Higgs mass in supersymmetric models. Supersymmetry and unification of fundamental interactions. Proceedings of the 12th international conference, SUSY 2004, Tsukuba, Japan, June 17-23, 2004 (2004), pp. 757-760. arXiv:hep-ph/0409073

29. A.D. Medina, N.R. Shah, C.E. Wagner, A heavy Higgs and a light sneutrino NLSP in the MSSM with enhanced SU(2) D-terms. Phys. Rev. D 80, 015001 (2009). arXiv:0904.1625

30. R. Huo, G. Lee, A.M. Thalapillil, C.E. Wagner, $S U(2) \otimes S U(2)$ gauge extensions of the MSSM revisited. Phys. Rev. D 87, 055011 (2013). arXiv:1212.0560

31. A. De Simone, J. Fan, M. Schmaltz, W. Skiba, Low-scale gaugino mediation, lots of leptons at the LHC. Phys. Rev. D 78, 095010 (2008). arXiv:0808.2052

32. M. McGarrie, General gauge mediation and deconstruction. JHEP 1011, 152 (2010). arXiv: 1009.0012

33. R. Auzzi, A. Giveon, S.B. Gudnason, T. Shacham, A light stop with flavor in natural SUSY. JHEP 1301, 169 (2013). arXiv:1208.6263

34. R.T. D'Agnolo, E. Kuflik, M. Zanetti, Fitting the Higgs to natural SUSY. JHEP 1303, 043 (2013). arXiv:1212.1165

35. N. Craig, S. Dimopoulos, T. Gherghetta, Split families unified. JHEP 1204, 116 (2012). arXiv: 1203.0572

36. K. Blum, R.T. D'Agnolo, J. Fan, Natural SUSY predicts: Higgs couplings. JHEP 1301, 057 (2013). arXiv: 1206.5303

37. M. McGarrie, Hybrid gauge mediation. JHEP 1109, 138 (2011). arXiv:1101.5158

38. H. Abe, T. Kobayashi, N. Maru, K. Yoshioka, Field localization in warped gauge theories. Phys. Rev. D 67, 045019 (2003). arXiv:hep-ph/0205344

39. L. Randall, Y. Shadmi, N. Weiner, Deconstruction and gauge theories in AdS(5). JHEP 0301, 055 (2003). arXiv:hep-th/0208120

40. A. Falkowski, H.D. Kim, Running of gauge couplings in AdS(5) via deconstruction. JHEP 0208, 052 (2002). arXiv:hep-ph/0208058

41. A. Djouadi, The anatomy of electro-weak symmetry breaking. II. The Higgs bosons in the minimal supersymmetric model. Phys. Rep. 459, 1-241 (2008). arXiv:hep-ph/0503173

42. J.R. Ellis, G. Ridolfi, F. Zwirner, On radiative corrections to supersymmetric Higgs boson masses and their implications for LEP searches. Phys. Lett. B 262, 477-484 (1991)

43. J.L. Lopez, D.V. Nanopoulos, New theoretical lower bounds on the Higgs sector of minimal SUSY. Phys. Lett. B 266, 397-402 (1991)

44. M.S. Carena, J. Espinosa, M. Quiros, C. Wagner, Analytical expressions for radiatively corrected Higgs masses and couplings in the MSSM. Phys. Lett. B 355, 209-221 (1995). arXiv:hep-ph/9504316

45. H.E. Haber, R. Hempfling, A.H. Hoang, Approximating the radiatively corrected Higgs mass in the minimal supersymmetric model. Z. Phys. C 75, 539-554 (1997). arXiv:hep-ph/9609331 
46. G. Degrassi, S. Heinemeyer, W. Hollik, P. Slavich, G. Weiglein, Towards high precision predictions for the MSSM Higgs sector. Eur. Phys. J. C 28, 133-143 (2003). arXiv:hep-ph/0212020

47. K.R. Dienes, E. Dudas, T. Gherghetta, Extra space-time dimensions and unification. Phys. Lett. B 436, 55-65 (1998). arXiv:hep-ph/9803466

48. A. Abdalgabar, A.S. Cornell, A. Deandrea, M. McGarrie, Large $A_{t}$ without the desert. JHEP 1407, 158 (2014). arXiv:1405.1038

49. A. Arvanitaki, G. Villadoro, A non standard model Higgs at the LHC as a sign of naturalness. JHEP 1202, 144 (2012). arXiv: 1112.4835

50. K. Blum, R.T. D'Agnolo, 2 Higgs or not 2 Higgs. Phys. Lett. B 714, 66-69 (2012). arXiv:1202.2364
51. R.S. Gupta, M. Montull, F. Riva, SUSY faces its Higgs couplings. JHEP 1304, 132 (2013). arXiv:1212.5240

52. S. Dawson, A. Gritsan, H. Logan, J. Qian, C. Tully, et al., Higgs Working Group Report of the Snowmass 2013 Community Planning Study. arXiv: 1310.8361

53. D. Asner, T. Barklow, C. Calancha, K. Fujii, N. Graf, et al., ILC Higgs white paper. arXiv:1310.0763

54. M.E. Peskin, Estimation of LHC and ILC capabilities for precision Higgs boson coupling measurements. arXiv:1312.4974

55. S. Kanemura, K. Tsumura, K. Yagyu, H. Yokoya, Fingerprinting non-minimal Higgs sectors. arXiv:1406.3294 